\title{
Looking at Islamic Patterns II: Making Sense of Geometry
}

\author{
Peter R. Cromwell \\ 28 July 2021 \\ http://girih.wordpress.com
}

\begin{abstract}
Islamic geometric patterns are arrangements of interlocking stars and polygons. Why do they attract our attention? Following Silvia's model, interesting stimuli are hard to process yet comprehensible. To understand what it means to make sense of a geometric pattern we explore representations of space and geometric structure. Perceptual and linguistic evidence yields small sets of primitive objects and qualitative relations that suffice to build a synthetic geometry of perceptual space; constructing patterns requires the capability to divide lines and circles into equal parts, but does not rely on measuring lengths or angles explicitly.

We compare eight representations of a star motif that demonstrate different approaches, different spatial frames of reference, and different levels of abstraction. A representation may be parametrised to represent a category, which allows us to verify that it captures salient features (the object should not be an anomaly in the category). We conclude that sequential constructive representations (algorithms) do not provide a good model for spatial structure, and that compression (encoding) and comprehension (making sense) are distinct.

Chunking and schemas capture generic structure in unfamiliar contexts, in particular repetitive, composite, modular and hierarchical structure. The large corpus of Islamic patterns exhibits constant innovation over hundreds of years. We give examples to illustrate a clear trend towards increasingly complex structure, of both modular and hierarchical forms.
\end{abstract}




\section{Introduction}

Why do we look at geometric patterns? What attracts us, gives us pleasure, and retains our interest? This is the second of two papers that explore what geometric patterns tell us about the working of the human visual system, and which properties of Islamic geometric patterns make some more successful than others. The two papers refer to each other to make connections, but can be read independently. The first paper [35], which we shall refer to throughout as part I, focussed on low-level features of patterns that are processed fluently, and noted that they correspond to non-accidental properties such as edges, corners, straightness and alignment. We also noted that the visual system identifies affine relationships like collinearity, parallelism and convexity more accurately than metric properties based on lengths and angles, and we illustrated how this can be exploited to create patterns with illicit but convincing combinations of geometrically incompatible motifs.

Patterns that activate low-level feature detectors are pleasing to look at, but we soon tire of them. In this paper we consider how the knowledge and experience of the viewer influences the interpretation of the data, and what the pattern designer can do to develop patterns with comprehensible spatial structure that will attract and retain the viewer's attention.

This paper does not assume the reader has a background in either Islamic patterns or the psychological aspects of vision, As with part I, relevant ideas are introduced as needed. Cognitive psychology, the study of mental processes, has a wide range of applications. General introductions that discuss the foundations used here include [44, 61], and some of the other references are survey or review papers on specific topics.

The paper unfolds as follows. In the $\S 2$ we present a 3 -layer model of visual processing that highlights the gap between parallel processing for fundamental feature extraction and sequential processing for symbolic reasoning. Finding spatial structure is a multidimensional problem, and bridging the gap seems to be key to understanding how we make sense of geometric data. In $\S 3$ we consider what makes things interesting: while perceptual fluency brings pleasure, it is the lack of cognitive fluency, the inability to resolve data immediately into a comprehensible structure, that creates interest. In $\S 4$ we distinguish between the visual identity of landmarks and their spatial relationships, and note that a person's preferred processing style (visual or spatial) can affect their response to geometric data. In $\S 5$ we review strategies used to organise information, such as hierarchies of parts and abstraction, both of which reduce the load on working memory. In the next five sections we study aspects of representations, and explore which primitive objects and relations are important for encoding geometric patterns. We use the fact that parametrising a representation defines a category: when the represented data is a typical member of the category it gives us confidence that the representation has abstracted salient elements. Good representations are expected to be economical (small to store) and efficient (easy to process). In $\S 7$ we compare many representations of a simple motif, which exposes the fact that such properties are hard to define and quantify. We also note that the cost-benefit analysis for a representation should include its cost of production, and the value of its information to a consumer or end-user. All these elements are drawn together in $\S 11$ to assess what constitutes comprehensible structure in a pattern. Traditional Islamic patterns underwent constant innovation for over 600 years. The examples in $\S 12$ illustrate significant stages in the evolution of star patterns in Persia and Central Asia from the twelfth to the 
eighteenth centuries. This development shows a growing ability to overcome the inherent constraints of the geometric medium, and a sustained trend towards increasingly intricate yet decomposable structure, leading to patterns that are simultaneously more complex and more comprehensible.

\section{A mental model}

It will be useful in the following discussion to have a simple model that provides a framework for the basic perceptual and cognitive functions involved in visual processing. The three layers of the model are summarised in Figure 1: on the left are functions concerned with data collection and the extraction of general purpose, non-accidental features; in the middle layer relationships between the features are discovered, adding structure and context to produce information; on the right we become aware of relevance or meaning, and have knowledge. Flow through the model is accompanied by a large reduction in volume as data is distilled and enriched.

\begin{tabular}{|c|c|c|}
\hline $\begin{array}{c}\text { Signal/Data } \\
\text { Processing }\end{array}$ & $\begin{array}{c}\text { Information } \\
\text { Processing }\end{array}$ & $\begin{array}{c}\text { Knowledge } \\
\text { Processing }\end{array}$ \\
\hline $\begin{array}{c}\text { sensing } \\
\text { frequency tables } \\
\text { filtering } \\
\text { feature detection }\end{array}$ & $\begin{array}{c}\text { representation } \\
\text { implicit memory } \\
\text { chunks, schemas } \\
\text { metacognition }\end{array}$ & $\begin{array}{c}\text { understanding } \\
\text { explicit memory } \\
\text { attention } \\
\text { analysis }\end{array}$ \\
\hline parallel & multidimensional & $\begin{array}{c}\text { linear, serial } \\
\text { conceptual }\end{array}$ \\
perceptual & \multicolumn{2}{|c|}{ social, cultural adaptive, versatile } \\
iniversal & learns from experience \\
stimulus-driven & limited by working memory capacity \\
\hline \multicolumn{2}{|c|}{ initiated automatically } & deliberate \\
subconscious, inaccessible & conscious, aware \\
\hline
\end{tabular}

Figure 1: A simple 3-layer model for visual processing.

In part I we applied elements of perceptual processing from the left half of the model. This included the physiology and psychophysics underlying layer 1 such as the innate signal processing that detects edges and corners, and further filtering for detecting alignment (parallel or collinear lines) and imaginary features such as mirror lines and rotation centres. The boundaries between the layers are blurred. Perceptual organisation straddles layers 1 and 2: fixed processes such as grouping by built-in features (colour, orientation, texture, proximity), short-range interpolation between line segments to bridge small gaps, and grouping segments that lie on a smooth line (good continuation) fall in layer 1, whereas adaptive processes that discover task-specific regularities to identify higher-order assemblies and configurations like stars and more abstract properties such as symmetry types belong to layer 2. In this paper we investigate some of the cognitive processes in layer 2 .

The three layers use memory in different ways. In the data layer, memories hold statistical properties of the environment. These frequency tables are either innate or trained very early in life through exposure to visual data. Implicit memories [58] are formed from cumulative associations, and include procedural knowledge and skills (such as playing a musical 
instrument). These rules and heuristics learned from past experience can produce biased interpretations, an effect that can be exploited to create visual illusions. Implicit memories are hard to verbalise and feel like intuition. They are also difficult to unlearn and replace. Explicit memories can be brought into conscious awareness in layer 3 and include declarative knowledge and episodes of autobiography. They are accessible for verbal description, and hence can be passed on quickly to others. They can be updated and overwritten.

The metacognitive processes in the information layer are an important feature of the model. They provide a mechanism for the brain to monitor and regulate the performance of other processes. When we become alerted to processing difficulties through feelings of uncertainty or confusion, or a realisation that we do not know something, we can focus attention and resources on the problem. The fluency hypothesis, that successful processing is rewarding, is also a consequence of this mechanism. We applied it in part I, noting that recognising regularities in data is pleasing, and we shall see further examples later.

The knowledge layer performs the high-level functions associated with conscious thought: analysis, reasoning, labelling, planning and problem solving. Many of these functions depend on the use of symbols or language.

Flow through the system is mostly from left to right, from sensing to understanding. However, low-level functions can be modified by top-down control signals. For example, focussing attention on items for analysis or exploration at the conscious level needs access to working memory and internal representations, and possibly tunes low-level filter parameters.

Processing becomes increasingly subjective from left to right with dependencies ranging from the frequency of features in the environment, to the variety of the observer's experience, to beliefs and cultural influences. It would be convenient to assume that visual processes are universal, that the physiology and innate processing that supports vision has not changed for thousands of years and that, in particular, we see in the same way as the medieval people who created traditional Islamic patterns. Experiments in cultural psychology (see [88] for a survey) have shown that high-level processes such as reasoning strategies are influenced by social and cultural practices. At the lower levels, growing up in a 'carpentered environment' surrounded by forms composed of straight lines and right angles makes you more susceptible to some visual illusions. However, for the topics considered in this paper, we shall assume that we respond to geometric patterns in the same way as the people who made them.

\section{$3 \quad$ What makes things interesting?}

Our attention is naturally drawn to things that are surprising or puzzling. We focus on things that are unfamiliar, rare, unexpected, unconventional, incongruous, contradictory, ambiguous or indeterminate. These properties are largely subjective, being associated with something that has not been seen before, has not been seen in its current context, or that creates some degree of uncertainty. Such disruptive stimuli are hard to process.

The fact that data is hard to process is actually a very useful message. It could trigger a metacognitive alert to signal 'this is odd - you should pay attention to it'. If the visual system is adaptive and learns new processing strategies when it encounters data structured in novel ways then the hard-to-process message also provides an elegant method for detecting novelty or the unexpected: we do not have to search our memory for evidence that we have seen something before - we merely note that we have not learned how to process it yet.

The hard-to-process signal is one of the components in Silvia's appraisal model of interest 
[103, 104, 105]. Appraisal theory [42] asserts that emotions are induced by the evaluation (appraisal) of a situation. The situation can be sensed, remembered or imagined, static or dynamic. Something about the situation will trigger an appraisal, and it will be assessed for importance, relevance, and potential implications; if there is uncertainty about what is happening, there will also be an assessment of the ability to cope and be in control. Appraisal processes are metacognitive (fast and automatic). Appraisals assess a variety of criteria, and each emotion is associated with a unique combination of them. Because it is the appraisal that induces the emotion, not the situation itself, different situations can lead to the same emotion. Appraisals are subjective, so different people may evaluate the same situation differently and consequently feel different emotions. Moreover, the same person may evaluate a situation in different ways and feel different emotions at different times.

Silvia's model of interest has two criteria: the situation must be hard to process, and it must be comprehensible. The first criterion means that the data contains elements that are surprising, novel or complex. This creates uncertainty, and the second criterion is an assessment of coping potential: what is the likelihood that the data can be made coherent?, what resources are available to make sense of it?, how steep is the learning curve? If both criteria are matched then we feel interested and act to explore the situation further. The activity will stop when we understand what is going on or when we admit defeat and accept that insufficient progress has been made to justify continuing. In both cases we no longer find the situation interesting. The latter outcome induces negative emotions: confusion, disappointment, possibly annoyance that we have invested resources that could have been used on something more beneficial. Finding a solution has several consequences. First, successful processing produces a reward (an 'Aha' experience). Second, it means we have learned a new skill: we understand the situation so that, if it arises again, it will not be hard to process and we will not find it so interesting in future.

The variability of the emotional response to a given situation depends not only on experience, but also on personality. Different people will weight the relative importance of the appraisal criteria differently. Experiments on interest [106] found that, for about two-thirds of people, novelty and complexity have a stronger effect on interest than coping potential, while the factors were reversed for the other third. The two classes also correlate with the openness personality trait (curious or cautious) with people in the larger class having higher openness.

How does this apply to geometric patterns? Suppose that looking at a geometric pattern provokes the hard-to-process signal. For some people the preliminary triage to assess relevance will produce negative results (too abstract, only a pattern), leading to the conclusion that the event is unimportant, and hence the interest response is not induced. The more curious viewers who get past this point then have to assess whether the pattern is comprehensible. It is unclear how to do this. To comprehend something is to explain and predict its behaviour, to identify underlying causes, to grasp its significance and implications, and to develop actions to deal with it. For patterns we want to discover meaningful (non-accidental) relationships in the data and rules that govern its structure, and possibly the thought processes of the designer.

The pleasure-interest model for aesthetics (PIA) [54, 55] also employs a two-stage framework. The first stage, known as default processing, comprises the objective data processing of layer 1 plus the subjective information processing of layer 2 based on what the viewer already knows. Thus, the fluency of default processing depends on properties of the data 
and the experience of the viewer. When the default processing is dysfluent, and the viewer is open to cognitive enrichment, a second, optional, stage tries to reduce the dysfluency (make sense of the data). An insight that reduces the dysfluency generates an 'Aha' reward that is distinct from the pleasure derived from fluency.

Both models agree that dysfluency in default processing indicates that something interesting has happened, which triggers further cognitive processes to try to make sense of the problematic data. However, both descriptions are incomplete. In Silvia's model we do not know how to assess coping potential before starting work. Similarly, in the PIA model dysfluency reduction is a sign of successful coping, but we do not know how to measure dysfluency. Neither model explains what leads us to expect that coherent structure can be found and that effort should be invested.

The more we find rules and relationships in data, the more predictable it becomes, and the less information we need to represent the structure and recreate it. This observation led Chaitin to remark "comprehension is compression" [24]. We shall see later that this oversimplifies the problem, but it is a useful place to start. Compression and interest are key elements in Schmidhuber's curiosity framework [99, 100]. The data layer in Figure 1 extracts commonplace regularities, but we need further processing to find structure among these fundamental features, something more flexible that discovers and learns new, higher-

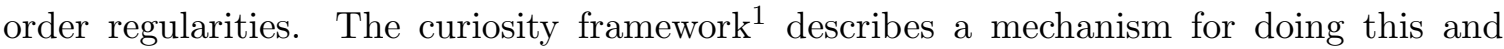
consists of the following components:

- Compression: A process uses experience from previous examples to find relationships between elements in the data. This structural organisation of parts produces a more compact representation of the data. The process may overfit the data, finding structure that is not justified.

- Curiosity: The relationships used by the compression process are empirical principles that have been found in historic data. There is a drive to discover new regularities within the data stream to provide incremental updates to the compressor and improve its capability. Although, in theory, the compressor can exploit any regularities in the data, some of them may not be detectable.

- Interest: Searching for new regularities to improve the compressor could continue indefinitely, so we need to monitor progress. The discovery of a new regularity generates a reward - the significance of the discovery and the size of the reward are determined by the reduction in size of the output representation. The reward is for the intrinsic benefit of learning; it is not dependent on attaining any immediate practical benefit.

When data is already highly compressible, we get no reward. Similarly, while the data remains incompressible, we get no reward. The data is only interesting while we anticipate it contains unknown yet learnable regularities. Curiosity is driven by the desire to maximise the reward.

In this form, the curiosity framework focusses on relationships within a single stimulus. It has been extended $[57,97]$ to take account of the benefits of finding coherent structure

\footnotetext{
${ }^{1}$ This framework was motivated by research in artificial intelligence and applied to algorithms for exploratory machine learning in which the measure of progress (interest) is defined as the rate of change of compression ratio as a learned compression algorithm evolves. Schmidhuber has also applied the same model to problems in human psychology.
} 


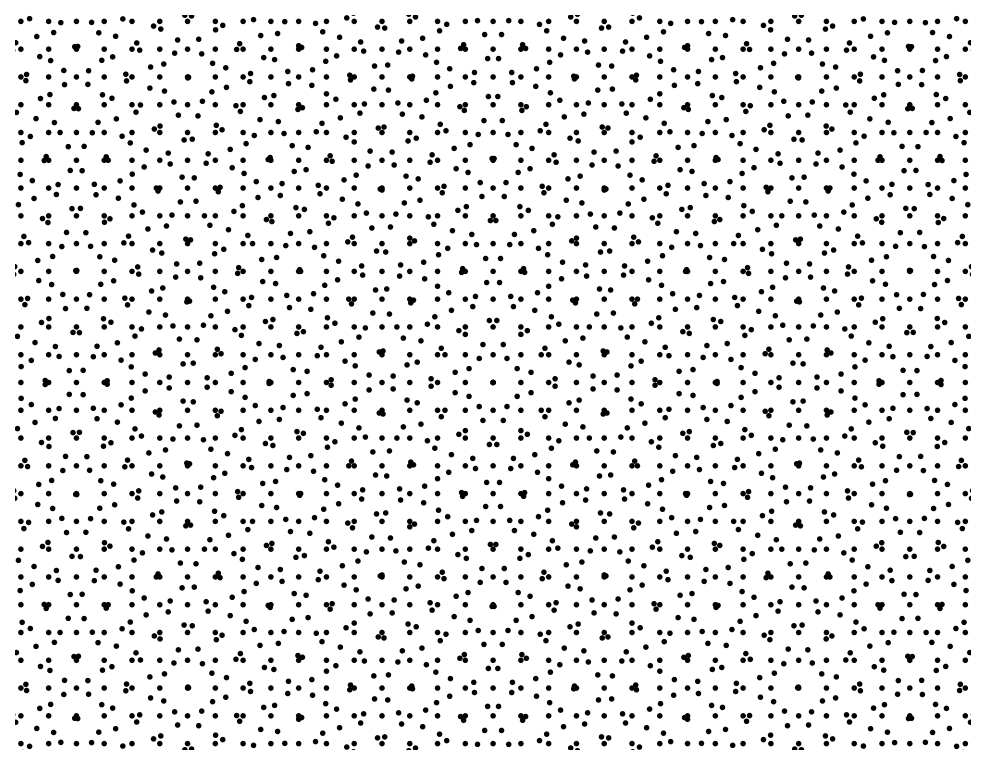

Figure 2: Arrangement of dots formed by overlaying three square lattices rotated at $120^{\circ}$ intervals. Example from [81, Fig. 7].

shared by multiple items, a process that generates a reward for recompressing stored data in a more economical form.

We can illustrate the ideas in this continual improvement process using the dot pattern in Figure 2. It has a simple global structure that we are unable to find: it is formed from three square lattices of dots overlaid at $120^{\circ}$ intervals. However, the urge to find structure is very strong, and configurations of closed symmetric shapes (familiar, regular local structures) appear and disappear among the dots. Even though the search for a stable, coherent, large-scale structure is inconclusive, the series of partial successes helps retain our interest as we feel we ought to be able to make sense of it.

Chater and Loewenstein [28] have also asserted that we have an innate drive to find simple structure in data, that we have a preference for comprehensible stimuli, and find it rewarding to make sense of them. In the following sections we shall explore what makes a geometric pattern hard to process, and what kinds of structures are comprehensible.

\section{Visual and spatial information}

When we look at a geometric pattern, we are performing two kinds of processing: visual processing concentrates on objects and their inherent properties (size, shape, colour, identity), and spatial processing concerns location, relationships, and motion. These are colloquially known as the What and Where processing streams, and correspond to different pathways in the brain [16]. The following examples illustrate the differences.

When we are in our home town, we have a good understanding of its layout: we know how places are situated in relation to one another, we know the connections between them, and we can plan routes that we have not taken before. When we visit an unfamiliar town, we have none of this spatial knowledge, yet we can still navigate successfully by using 
visual markers: "continue straight ahead, turn right at the post box, go over the railway bridge, then left at the church". We do not need to know the location of anything, we just have to spot landmarks. Ideally, a landmark should be conspicuous (easy to detect in the landscape), unmistakable (easy to recognise ${ }^{2}$ ), invariant (not change with the seasons), and unique in the local area. If a landmark is asymmetric or has a single vertical plane of mirror symmetry, it also has a definite orientation, which determines a unique reference direction.

Unlike higher cognitive abilities such as language and reasoning, navigating in our local environment is an ancient skill we share with non-human animals. Like us, animals have distinct visual and spatial strategies (see [112] for a survey). For example, Beewolf wasps locate their burrows using nearby landmarks, and they make reconnaissance flights of the local area to enable them to return home. Varying the configuration (circle or triangle) and the visual identity (pine cones or stones) of the objects forming the landmark array showed that the spatial arrangement was more important than the identity of the objects $[110,111,114]$. However, it is not always obvious which strategy is being used. Cartwright and Collett $[19,20]$ trained honeybees to locate food relative to a single cylindrical landmark in an otherwise featureless environment. This task appears to involve spatial processing. However, when the size of the cylinder was halved or doubled, the bees searched proportionately closer or further away, showing that they derived a sense of scale from the landmark. One hypothesis is that the bees store a snapshot image of the landmark viewed from the reward site, and relocate the reward visually by comparing their current view with the stored image and moving around until the views match.

Spatial information is about more than navigation. We can touch and manipulate objects in the environment, an interaction that provides strong sensor-motor feedback about the spatial relationships between things. Even though uncertainty about locations is inherent in the visual system, this opportunity to test our interpretation makes us disproportionately confident about spatial relationships [47]. Spatial awareness is not just visual: we can locate the direction of auditory signals, and build a cognitive map from verbal or written descriptions. While language and reasoning operate with one-dimensional chains of words and symbols, spatial information deals with multidimensional structures (diagrams, plans, networks and hierarchies). It also facilitates the mental rotation of objects so they can be recognised from multiple viewpoints.

Spatial information can be presented in different formats. Two approaches to navigation can be described as path-based and map-based. The path-based approach evokes a narrative of a person moving through the environment, noting salient landmarks and routes between them. It is sequential, dynamic, and egocentric - the current position and orientation of the perceiver provide the frame of reference. The map-based approach is like an aerial view in which everything is presented simultaneously. It is non-linear, static, and allocentric the frame of reference is provided by the environment, and objects are related to each other in configurations that are independent of the location of the perceiver. When information is presented in one format and queried in the other, we can change our stance, but there is a delayed response as we perform the conversion [83]. We may use both formats in parallel and select our stance depending on the task being performed [17].

People differ in the ways they represent and process spatial information. The development of spatial representations in young children progresses through three stages: use of

\footnotetext{
${ }^{2}$ The landmark types need to be familiar to the visitor: "turn left at the mosque" does not help if you do not recognise one when you see it.
} 
isolated visual landmarks, paths connecting sequences of landmarks, and abstract structures with more complicated interconnections [102]. As adults, we may favour one of these methods, perhaps preferring verbal descriptions and landmarks to abstract configurations. Such preferences define groups of people who pay attention to different features, build spatial knowledge in different ways, and perform differently on tests of spatial ability [91]. These groups are correlated with gender, the average male score being higher than the average female score. Of the cognitive abilities that exhibit a gender difference, spatial ability consistently shows the biggest difference. This is a cross-cultural effect, and probably has a mix of biological and social causes $[23,94]$, but there is evidence that gaming can improve spatial skills and the widespread playing of video games may reduce social factors that contribute to the difference [107].

Geometric patterns function on a smaller scale than the navigation examples we have used so far, but spatial processing applies in the same way - it concerns relationships between identifiable parts. The primary constituents of a pattern, the individual lines and shapes, do not have sufficient weight to form suitable landmarks on their own. However, perceptual organisation groups elements into larger symmetrical units such as star and rosette motifs. We remarked above that ideal landmarks are conspicuous, unmistakable, unique visual markers in the landscape. First, note that Islamic star patterns are produced by repeating a template (see part I), hence no element in a pattern is unique. From a geometric point of view, local and global centres of rotational symmetry are the natural candidates for landmarks as they are well-defined and isolated from one another. Global rotation centres are defined by long-range relationships without an associated localised visual cue - they are pure spatial information. Fortunately, in many patterns, star motifs (local centres) are positioned to coincide with global centres, which provide conspicuous cues to the location of the global centres.

Just as in the natural world where physical properties like the magnetic field and gravity define the reference directions North and vertical, which are independent of any landmarks, the border of a decorative panel defines (quite literally) an extrinsic frame of reference for the pattern. In theory, a panel shows an arbitrary finite portion of a pattern that repeats indefinitely in all directions. In practice, Islamic panels contain a whole number of repeat units and the border coincides with mirror lines of the pattern. Furthermore, panels are often quartered with vertical and horizontal mirror lines passing through the centre. Since the intersection of two mirrors must be a global rotation centre, the natural reference points defined by the frame (its corners and centre) also coincide with landmarks of the pattern.

Visual and spatial characteristics can enhance or compete with each other. Figure 3 shows three geometric patterns whose compartments have been embellished in various ways. The side-lighting on the embossed bronze door in (a) reveals raised rings of teardrops encircling a dome, which reinforce the local rotation centres in the pattern, and thus amplify the landmarks. The interlaced ribbons in (b) are carved into stone blocks. Single flowers have been added to many of the compartments, but they are evenly distributed rather than occupying a few judiciously selected compartments as in (a) so they do not emphasise the landmarks. The flowers themselves are rather simple, generic rose-like motifs and do not draw attention - they merely balance the austerity of the geometric figure. The wooden door in (c) is assembled from interlocking parts slotted together. The inserts that fill the compartments are carved with foliate arabesque and stylised lotus buds; the high level of detail in the arabesques starts to distract from the pattern. In more elaborate examples the 


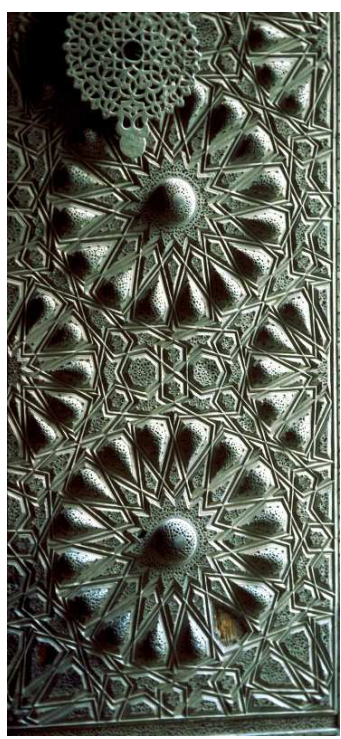

(a)

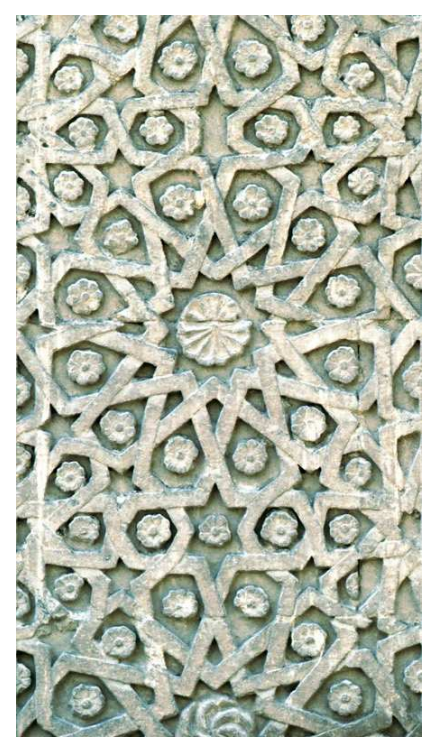

(b)

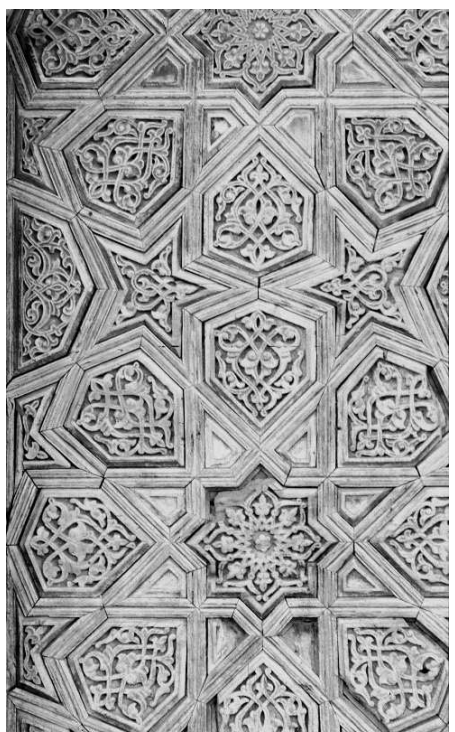

(c)

Figure 3: Attention may be drawn to landmarks or diverted away from them by other features of a design.

visual elements dominate and the pattern plays a secondary role, providing a framework of compartments to house panels of naturalistic floral ornament.

\section{Organising information}

How our visual system makes sense of data is not fully understood. In part I we explored perceptual organisation, the subconscious processes that give low-level structure to sensory data. These include the biochemistry and psychophysics of the retina and early visual system, and empirically observed rules like the Gestalt principles of grouping and interpolation. We also identified regularities in the data (features that are processed fluently) with non-accidental features of the environment. This processing is universal and fixed (although it may be fine-tuned), and has sufficient capacity to keep up with the data rate. However, finding higher-level structure requires something more adaptable.

Cognitive processing relies on past experience and hence on memory. While long-term memory is usually regarded as unlimited, working memory has low capacity, which imposes severe constraints on the number of elements that can be used simultaneously. Two strategies for organising information reduce the processing load: break down a specific instance into a hierarchical structure of nested chunks [51], each small enough to be considered as a whole, or summarise the essence of typical situations into generic abstract schemas [60]. It is not clear how chunks and schemas are acquired and maintained, but predictions based on the hypothesis that they exist are consistent with experiments.

Working memory is a temporary store for manipulating information. By giving people small concurrent tasks and noting which ones interfere with each other, it is possible to show that working memory has several independent specialised subsystems. In the Baddeley- 
Hitch model [5] these include separate processing for aural-verbal stimuli and visio-spatial stimuli. The visio-spatial processing may be further divided into components for visual tasks, path-based and map-based spatial tasks, and 3D transformations such as rotating small objects $[78,79]$. Although the number of items that can be processed simultaneously varies slightly, it is always very small - measured in single digits. It depends on the task, the person, and possibly a trade off between the number of items and the level of detail.

Miller [85] drew attention to the limits of working memory and introduced the process of 'chunking' information as a way around it. A chunk is a small collection of things that are closely related. While chunks may not have accessible names, they can contain references to each other, so a hierarchy of nested chunks can be formed and tasks split into manageable pieces. When chunks are recalled from memory, each is delivered whole, but the order in which its members appear may vary in repeated recalls.

Much of the early work on chunking studied chess players [52]. In this context a chunk is a group of pieces in a configuration that has significance within the rules of the game. Pieces can act at a distance so pieces in a chunk need not be in close proximity - the chunk may be based on functional relationships like attack and defence. Pieces can serve multiple purposes and chunks can overlap. A chunk need not retain full detail: the fact that a square is occupied or empty may be sufficient. A chunk has an absolute frame of reference for its location on the board: identical local arrangements of pieces in different places result in different chunks. One of the key findings is that chunking depends on the skill and experience of the player. A board position does not exist in isolation, but is a snapshot in a sequence of unfolding strategies; the rules of the game give salience to certain configurations, and experts are better at exploiting this semantic structure.

A schema records the essence of a class of things or events rather than details of any particular instance. By convention, the use of a schema is indicated by small capitals. For example, TREE is a generic abstract representation of a large class of tall plants with woody stems. The terseness of the abstraction can lead to vague membership criteria: what about cork, bamboo, banana or bonsai? Besides its defining characteristics, a schema also carries stereotypical attributes that can be supplied in the absence of information about a particular example: a TREE has a trunk that is suitable for timber. This property has significant consequences for how we interpret data, leading us to see what is expected, not what is actually there. Pattern designers can turn this effect to their advantage. For example, the STAR schema comes with an expectation of radial symmetry and, if there are no cues in the pattern to inhibit this interpretation, we see perfect stars. However, this source of bias has serious implications in other contexts.

$\begin{array}{lll}\text { THING } & \text { CONTAINER } & \text { MATCHING } \\ \text { LOCATION } & \text { PART-OF } & \text { PATH-TO-GOAL } \\ \text { PATH } & \text { GROUP } & \text { SPACE } \\ \text { BOUNDARY } & \text { REPETITION } & \text { VERTICAL } \\ \text { CONTACT } & \text { SEQUENCE } & \text { STRAIGHT } \\ \text { OBSTRUCTION } & \text { CYCLE } & \text { CENTRE }\end{array}$

Figure 4: Fundamental schemas that arise from sensory-motor experience.

The most primitive schemas are based on simple structures that recur in our everyday

\footnotetext{
${ }^{3}$ This is analogous to trading off the number of photographs that can be stored in a camera against the resolution (number of pixels) or quality (bits per pixel) of the photographs.
} 
experience and are grounded in sensory or motor interaction with the environment (see Figure 4). Before infants can focus their eyes, they can track objects in motion and register a change of state: start, stop, disappear. The earliest schemas we learn as preverbal infants are all spatial [80]. A THING (a nameless object without identity) changes LOCATION, following a PATH. It has a BOUNDARY that may come into CONTACT with something else. Objects may also act on each other in various ways: an OBSTRUCTION inhibits the motion of the object we are watching; an object can be placed temporarily in a CONTAINER, which supports, transports, and partially obscures it. Once we can recognise objects, we also discover relationships between them and can see them as a GROUP or a SEQUENCE. Whether the PART-OF relation is an innate schema that we impose to make sense of the world or something we abstract from structures in the world is a philosophical problem - in either case, it is indispensable for organising information. We also acquire more narrowly focussed schemas like VERTICAL and STRAIGHT, and more complex schemas that allow us to plan a PATH-TO-GOAL, understand CAUSE-AND-EFFECT, and compose scripts for situations. Universal schemas such as these form the foundations of conceptual thought and language.

When we encounter a new domain, we need to acquire new schemas to make sense of the unfamiliar data. Many of these are socially or culturally mediated and can be built on existing schemas. They become progressively more specialised the more we study the data. The PATtern schema is universal and based on RePetition. People who are culturally aware may also recognise GEOMETRIC patterns with non-figurative motifs composed of straight lines, the INTERLACING stylistic treatment of lines as interwoven ribbons, and even ISLAMIC patterns with interlocking stars and polygons. It takes training and time to develop the experts' schemas for art historical attributes based on period, region or style, design methods such as ADDITIVE or MODULAR, or technical attributes of geometric structure like the Bravais lattice that forms the underlying GRID of translations.

Analysis in terms of chunks and schemas has been applied to drawings and diagrams of various kinds. A circuit diagram is a representation of an electrical system that shows the components and their interconnections. The types of component are indicated by standard symbols; the arrangement of lines and symbols is topological and need not correspond to the positions of wires and components in a physical device. In operation, currents and signals will flow through the device and its internal state may change over time. None of the state information is recorded in the diagram, but the flow should be reflected in its structure. Meaningful configurations of symbols are constrained by physical laws, and the chunks produced by experts correlate with functional units in the circuit [41]. However, when symbols were arranged at random to produce unrealistic diagrams, experts were no better than novices at recalling the diagrams. Experts could also assign groups of symbols to functional categories (amplifier, rectifier) and use schemas to fill in expected components.

The Rey-Osterrieth figure is a line drawing used to assess visuo-spatial abilities by asking people to copy or recall it. The abstract configuration of lines does not encode information, there are no domain-specific rules governing the arrangement, it does not require specialist skill to interpret conventions or symbols and reveal its meaning, it is not designed to be attractive. It does, however, have a hierarchical structure and a natural division into frames and fillers. This structure facilitates chunking [89], the chunks follow the Gestalt principles of grouping, and the typical order of recall is to establish the skeleton that provides a framework for the other elements, add internal then external parts, then refine the details in each part. Although the figure is geometric, recall is easier if something like a FISH 
schema is used as a mnemonic.

How do we find structure and organise the information in an Islamic pattern? Two primitive schemas provide starting points: PATH and CENTRE. The first is a very early schema, which may explain the urge to follow the lines in a pattern. However, the paths that arise by travelling straight ahead at every crossing are often emergent properties of the pattern and not informative about its structure. A CENTRE of radial symmetry is a structural feature that has its origins in biological or physical processes that cause growth from a point (flowers or ripples for example). In a pattern, centres can be local or global symmetry elements of the pattern. The high-order centres stand out as important landmarks but, unfortunately, they also provide limited structural information. Take a sequence of nested neighbourhoods of a centre until you can no longer expand it without breaking the symmetry - this identifies a symmetrical motif of maximal size that we can call a rosette. Rosettes are strong non-accidental features that are islands of order, but they do not exhaust the pattern, and their arrangement is unexplained.

Moving beyond this is difficult because Islamic patterns do not have properties that make chunks easy to find. Rosettes make good chunks; they may overlap; the remaining shapes are diverse fillers. Chunking is driven by semantics or hierarchy, by function, purpose or PART-OF relations. The experiments on chess and circuit diagrams demonstrated that experts not only have more chunks gained through their experience with more examples, but they exploit semantic structure to produce deep hierarchies of nested chunks. When presented with random arrangements of pieces where the meaningful structure is absent, their advantage over novices almost disappears. A pattern is not an illustration or a symbolic diagram, it is not a tool to assist with reasoning, it is not one of a sequence of goal-oriented strategic positions. It carries no individual message, it has no functional parts. It has no semantic properties to support chunking.

What about hierarchy? Early patterns are composed by juxtaposition and extension of motifs, not by subdivision or assembly. This approach leads to a flat structure, not a hierarchy of parts. Even so, the repetition inherent in a pattern produces natural parts. As we saw in part I, mirror lines divide a pattern into repeat units. The lines connecting the CENTREs to their near neighbours also divide the plane into geometrically salient regions concerned with establishing the alignment and proportions of the motifs. Both annotations produce frames that can be used to locate elements of the pattern and define chunks. Applying Gestalt principles to the lines in the Rey-Osterrieth figure revealed its skeletal framework, but the annotations forming these frames are imaginary lines, not visible marks that belong to the pattern. These patterns are hard to organise, but this is what makes them interesting. However, if the underlying structure cannot be found, they are also somewhat disappointing. As we shall see in $\S 12$, later patterns display more overt forms of hierarchy.

\section{Representations}

So far, we have been using 'representation' as an undefined term, as though it is clear what it means. We have also assumed that it is easy to measure so that compression is meaningful. However, trying to clarify these issues touches on philosophical $[92,101]$ as well as psychological problems $[86,90,95]$. We do not know what mental representations are,

\footnotetext{
${ }^{4}$ Although there is no language of patterns and individual patterns do not carry particular messages, geometry and the geometric canon of ornament do have significance and meaning in the Islamic context [10].
} 
how they are acquired or stored, how they are grounded in the physical world or acquire meaning, nor how they relate to concepts and thought.

For our purposes, a cognitive representation ${ }^{5}$ is a mental construct that stores internal state and mediates between aspects of the physical or conceptual world and consequent thoughts or actions. It has value to any process that can decode and interpret the information held within it. A representation need only maintain a level of detail appropriate to its purpose. It could represent something imaginary. It could be inaccurate and misrepresent the true state of the thing it represents. It could be inconsistent and represent something impossible.

A representation has many layers of abstraction. For example, in a computer, information is organised in a data structure, whose fields have various data types, each type has a storage format as bits in memory, and bits are tied to charge in capacitors in a physical device. Our interest is at the higher levels — which elements are represented in the mind, not how they are instantiated in the physiology of neurons in the brain.

Let us make this more concrete with some examples. We can distinguish the following different kinds of representation.

- A description represents data as an unstructured list of category labels and attributes, forming explicit declarative knowledge. An art historical description of a geometric panel may include visual properties (size, shapes and motifs present, colouring, style, symmetry type) and also supplementary information about its location (building, town, region, position in the building, function of the building), age (date, period), people associated with its construction (architect, patron, who it honours), materials, assessments of condition, quality, importance, and so on.

- A diagram is a representation of structural relationships in the data. Maps of underground rail networks like the Tube in London are quintessential examples of structural representations. There are no visual landmarks to record, only the names of stations and the rail lines that run between them. There is also no need to preserve proximity, distance and orientation - topological properties (such as order of the stations) are far more important than geometric ones. The metric used by passengers is number of stops, not distance travelled. This means the layout can be distorted to make the map more legible: irrelevant details can be omitted, the lines can be smoother, and the stations arranged to prevent clustering.

- A logbook is an implicit representation of the environment, a record of a journey through the landscape that provides context. This accumulated wisdom can contain collections sampled from the raw data (such as a catalogue of chess positions), and summaries that form a representation of statistical relationships within the data. The summaries may be schemas for common structures, principles such as the rules of grammar or Schmidhuber's compressor, or heuristics such as those that give craftsmen an intuitive understanding of symmetry types.

- A simulation is a representation of functional relationships in the data. It is a dynamic model of objects and their interactions that allows us to make predictions

\footnotetext{
${ }^{5}$ Discussions of representations in psychology come in two common forms, depending whether the approach comes from below or above: either encodings of data in layer 1, or symbol systems that support processes in layer 3 (explicit memories, knowledge, language, predicate logic, and deductive reasoning). Although our focus will be on the former, the definition should accommodate both.
} 
and answer 'what if' questions. This kind of representation allows us to rotate objects in our mind's eye, or predict the track of a ball in motion and catch it. The purpose of the representation dictates which features are relevant: an orrery (clockwork model of the Solar System) illustrates the relative motions of the planets and their moons, but distances are not to scale.

- A recipe represents data as a constructive procedure - a set of instructions for recreating the data. It identifies fundamental parts and assembly instructions. The primitive objects, instruction set, and algorithm may vary according to the context. For example, the creative process used during the conception of a pattern will be in terms of motifs, and the rules used to establish their position, relative sizes, and alignment when they are assembled, whereas the procedure used to install the pattern on a wall will be a more Euclidean style point-joining construction of lines and circles.

Of course, these approaches - descriptive, structural, statistical, functional, constructive are illustrations. They are not intended as an exhaustive list, and cognitive representations can use a blended approach, combining different techniques as appropriate. Note, however, that latent properties (new properties that could be derived as logical consequences of the declared properties) are not part of the representation. For example, a description may be tabular, such as a list of distances between cities. If the distance of each city from at least three others is contained in the list then it is possible to reconstruct a map from the table that is unique up to orientation and mirror image. However, the table itself does not contain information about spatial configurations - it is not a spatial representation, just a list of facts. This does not preclude using a model for prediction since rules can be part of a representation.

An encoder is a process that creates representations. It provides a mapping between the objects and relations of the represented space onto those of the representing space. This may involve a change of medium (from sound pressure or light intensity to neural impulses) or a change of format (selecting and organising relevant features in the data). Encoding may be a data-driven process that has no knowledge of the current context or what the follow-on processes may do. It may create a fixed-format representation or it may write metadata into the representation so it can be interpreted and decoded (like column names in table, for example, or a legend for a graph, or compression parameters). The encoding process may be fully or partially reversible. If the representation is to function as an explicit memory then we need to be able to recover the most salient features of the data in enough detail. However, if its purpose is to trigger a follow-on action or process, the representation only needs to retain sufficiently many distinguishing features to reliably categorise the event. A hash function is an extreme form of this: it is fast to compute, small to store, fast to compare, may preserve some notion of similarity or proximity, but is irreversible. It can answer questions such as 'have I seen this before?' or trigger spontaneous responses that seem intuitive.

Let us now turn our attention to the representing space. What are the primitive objects (visual) and relationships (spatial) that constitute a representation of a geometric pattern? Since we regard representations as belonging to the middle layer of our mental model, we can approach this question from above and below.

Representations organise and summarise the outputs of layer 1. In our survey of psychophysical properties in part I, we noted that the geometry of perceptual space is far more 
attuned to affine properties of the data (straightness, collinearity, parallelism, convexity) than it is to metric properties (lengths and angles). We also found that alignment, centres of radial symmetry, and mirror lines are important low-level features.

Representations also support the explicit knowledge of layer 3. This gives us easy access to the conceptual, descriptive aspects of representations. For example, a star motif is a named category that could be defined by a checklist of other conceptual categories: spiky, symmetric, polygon. Each of these terms has its own such definition or is grounded in empirical examples. Even if these abstractions are well organised and have logically consistent dependencies, it is unclear how they reflect implicit features of any underlying representation.

However, layer 3 does provide a window onto non-verbal aspects of representations. A representation may not be directly accessible for conscious inspection in all its richness, but, as Landau and Jackendoff observed [71], any property you can talk about must be based on some element of the representation. They analysed language to extract all the purely spatial information it is capable of expressing. The ways of describing relative positions can all be derived from a few fundamental relationships: near, between, inside, around, in line with, in contact with. Notice that these are all qualitative relations. Unless we use a formal system of units, the continuum of distances are also specified through a small set of qualitative expressions, both allocentric (long, short) and egocentric (close, within arm's reach, far away). Even though these categories appear crude, we are able to represent absolute positions to high precision to facilitate motor control - we just cannot explain how.

Experiments with people who have no training in geometry, no language for geometrical concepts, and no experience with spatial tools like maps found evidence for a universal set of basic geometric concepts [38]. This core geometry includes points, lines, corners, connectedness, parallelism, right angles, squares and circles. Untrained people were less good at using metric properties such as detecting equidistance and symmetry, and found mental transformations like rotation very difficult.

These varied analyses provide a consistent account of what objects and relations make good candidates for primitive elements in geometric representations. Primitive objects are points, lines, corners (angles), and crossings, while relations are based on alignment (straightness, collinearity, parallelism) and a qualitative approach to relative positions and metric information. This may seem like a frugal starting point but, where psychology reveals what information is available, mathematics shows us this information is sufficient.

Plane geometry requires very little in the way of primitive objects and relations to lay its foundations. Points and straight lines suffice as primitive objects. The betweenness relation for three points creates what is known as ordered geometry. Ordered geometry contains collinear points and parallel lines, but it is not possible to prove that lines parallel to the same line are parallel to each other; adding parallelism as an equivalence relation creates affine geometry. Affine geometry does not include any mechanism to compare or transfer metric properties (so, for instance, you cannot describe a circle); adding two forms of congruence on line segments and angles as equivalence relations completes what we know as Euclidean geometry. Note that congruence facilitates comparison - it does not supply a unit of length or a coordinate system.

Although Euclid's account was held up as a model of rigorous abstract argument that is independent of diagrams, in fact, it relies on perceptually obvious properties of space. 


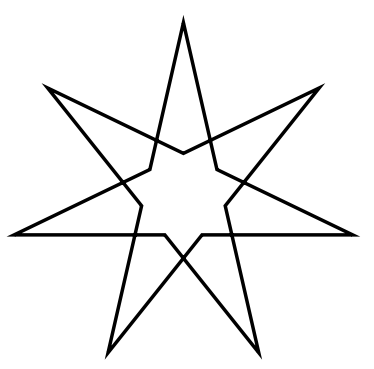

(a)

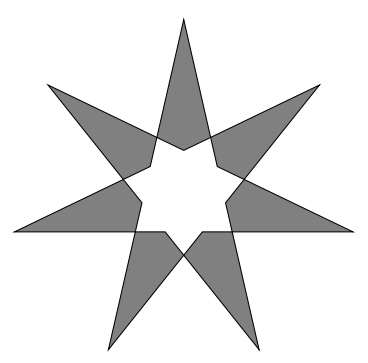

(b)

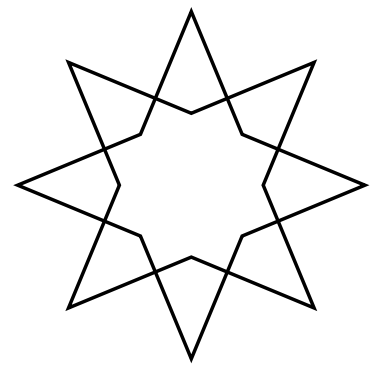

(c)

Figure 5: Star motifs.

These include: points are all the same, the plane is homogeneous (looks the same at any position) and isotropic (looks the same in any direction), lines are continuous and have no gaps, crossing lines have a point in common. Properties such as these were so obvious that they were not recognised as necessary axioms of geometry until the foundations of the subject were properly established in the late nineteenth century [63].

Choosing primitive elements and operations is tricky. Our intuitive understanding of the continuity of a finite curve demands that a line segment or a circle can be divided into $n$ equal parts for any integer $n$. One of the consequences of building Euclidean geometry on the properties of lines and circles is that we cannot perform all the basic operations we may expect. In particular, this constraint on permissible methods means that a circle cannot be divided into seven equal parts (something we shall need below) with the tools available. Our cognitive geometry must include such basic operations.

We shall now take the 7 -fold star motif shown in Figure 5(a) as a case study. Despite its simple appearance, it can be interpreted and represented in many ways, which will illustrate the difficulties in trying to decide what a representation ought to be. One of the first choices is whether the motif is composed of lines or shapes. The shading in Figure 5(b) highlights the interpretation of the motif as a ring of seven kites. The situation is analogous to the ambiguity of a standard figure that we discussed in part I: line-based or shape-based interpretations lead to perceptual organisations of the diagram as either two overlapping squares or two non-convex hexagons pushed together [35, Fig. 7]. The same choice is presented here. The similarity is even closer for the 8-pointed star motif shown in Figure 5(c) — is it two overlapping linear figures or eight connected kite shapes?

For the moment we shall pursue the line-based interpretation. The representation in Recipe 1 is a short algorithm that traces the star motif using turtle geometry. A turtle is a robot with a pen that draws a line as it moves around. It obeys a very limited set of instructions that allow it to move forward, turn on the spot, and raise or lower the pen. Thus, programming has an egocentric perspective, and all actions are relative to the current location and orientation of the robot - there are no absolute coordinates and no origin. The robot only moves in a straight line so it cannot draw circles or other curves.

Looking at the code, we can see that the robot travels a zig-zag path drawing equal length lines, turning alternately left and right through the specified number of degrees. The robot traverses the motif, starting at one of the outer corners of the star. However, some basic properties of the motif are not apparent from this representation. First, it is not 
clear that the start and end points of the path coincide. The angles are specified with high precision to try to ensure that this happens since any errors get amplified as the construction proceeds. Even if we discover that the zig-zag is a closed figure, we cannot immediately deduce that it has 7-fold symmetry without knowing the angle at the join. It is also not clear that the path crosses itself, or that some lines are collinear, and we do not know where the centre is.

Figure 6 illustrates two geometric constructions. The motif in Figure 6(b) is obtained by placing seven points equally spaced around a circle and drawing chords connecting every third point. Mathematicians regard this figure as a regular heptagon - seven sides of equal length, and seven equal angles — and it is called the regular star polygon of type $\{7 / 3\}$. The star motif, duplicated in Figure 6(a), is a subset of this figure obtained by deleting the middle segment of each line. This construction provides a causal mechanism that explains why some lines in the star motif are collinear.

Figure 6(c) illustrates a more direct construction that is comparable with diagrams found in medieval manuscripts containing Islamic geometric patterns. The process starts with a substrate, shown in red, consisting of two concentric circles and seven equally spaced radii. In part I this device with hub, rim and spokes is referred to as a wheel. The star motif is constructed by drawing a zig-zag line that bounces between the inner and outer circles where they cross the spokes. The mathematician's method has two parameters: the number of points and the step size, both of which are small integers ( 7 and 3, respectively). This craftsman's form also has two parameters, but is more flexible. The number of spokes (number of points of the star) is still an integer; the other parameter is the ratio of the radii of the inner and outer circles, which is continuously variable and controls the spikiness of the star. There is a unique value for the ratio that produces collinear lines in the star motif - the alignment is unexplained by this model and it appears to be a coincidence.

The important thing to notice is that the wheel is a representation of the star motif:

- it is a structural representation

- it is a simple model composed of a small number of primitive geometric elements (lines and circles)

- it encodes the relationships between the corners of the star motif by recording the linear and circular alignments between these key points

- it is a lossless encoding - the motif and its component parts (the corners, lines, crossings and kites) can all be recovered.

On its own, the wheel is unusable and has no value. The representation needs inter-

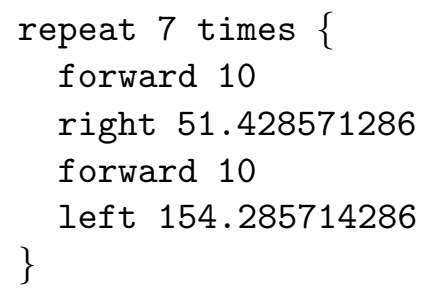

Recipe 1: Turtle commands to draw a zig-zag path. 


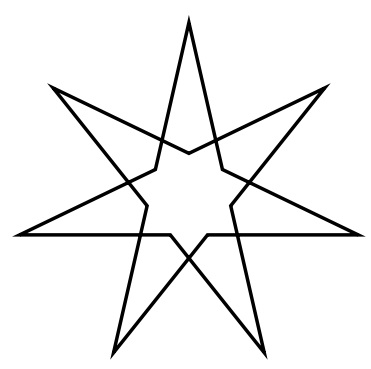

(a)

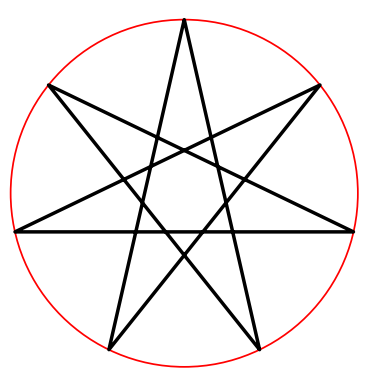

(b)

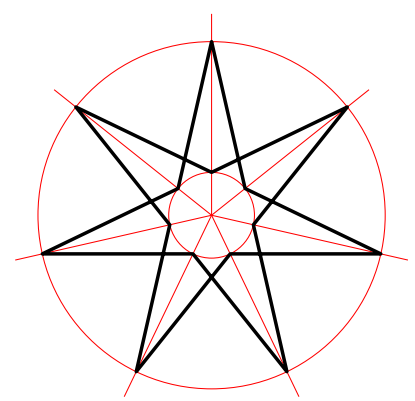

(c)

Figure 6: A 7-pointed star motif and two geometric representations.

preters or decoders to draw the star, obtain the centre point, compute the crossings, draw coloured kites, and so on. Its information is only accessible through decoders and, in this sense, the representation is encapsulated (to adopt terminology from the object oriented paradigm for software). Because of this, we should not consider a representation in isolation, but as part of an integrated system that includes the processes for interacting with it $[69,90]$. The encoder, representation, and decoder(s) are interdependent and must evolve together. The size of the representation and the complexity of its associated processes can be traded off against one another to satisfy resource limitations or speed requirements. We asserted above that latent properties are not part of a representation, but decoders can provide a mechanism to derive and access such properties. Notice that, from this perspective, line-based and shape-based interpretations of the star motif can be seen as different decoders with one underlying representation.

Compare the wheel representation of the star motif with the process that controls the turtle's behaviour. The algorithm in Recipe 1 is also a representation of the star motif:

- it is a constructive representation

- it uses a simple symbolic language composed of a small number of primitive commands

- it encodes the relationships between the corners of the star motif by recording the distances and angles between these key points

- it is a lossless encoding - it produces the motif.

This representation is self-contained and does not require a decoder (other than the robot). It is also inflexible and cannot do anything other than draw the motif. It stores no state, making it difficult to access properties such as the centre or circumcircle of the star. Positioning star motifs in a design needs control of properties like size, orientation, and alignment — properties that are more easily accessible from the wheel representation.

Recipe 1 expresses redundancy in the star motif as 7 -fold repetition in a loop, but this does not capture the true regularity of the star. The path followed by the robot is very sensitive to the angles in the algorithm. Changing the angle values will produce a drawing that probably will not close up, will not be symmetric, and will not look like a star. Even

\footnotetext{
${ }^{6}$ Contrast this with maintaining two representations and a translator as we saw in $\S 4$ for converting between parallel spatial models with egocentric and allocentric frames of reference.
} 
though the variants of the algorithm have exactly the same abstract form and complexity, the visual properties of the outputs are very different.

For economic reasons, it would clearly be useful if representations were not created as one-off bespoke items, but could be reused or shared. Different versions of a representation obtained by varying its parameters define a family of related forms with shared characteristics. In this parametrised form, the representation represents a category, not just an instance. To uncover what this category contains, we consider the representation as a generative model and vary its parameters [45]. In Recipe 1 we could take the lengths and angles to be parameters, but here we shall regard the lengths as fixed. Running the code with a range of angle values produces a family of equilateral zig-zag lines; the few symmetric polygons in the family are atypical and seem like accidents? The members of a category should not come as a surprise, and unknown members should be predictable from familiar examples. A square is a typical member of the regular polygon category, but is an exceptional member of the rectangle category. In the same way, the star motif is an exceptional member of the category derived from Recipe 1, and we therefore infer that the algorithm has not correctly abstracted the defining characteristics of the star.

What about the category generated by the wheel? The parameters of the wheel are: the centre and radius of the outer circle, the centre and radius of the inner circle, the origin from which the spokes emanate, and the directions of the spokes. The only constraints needed to ensure that the construction is valid are that the origin must be inside the inner circle, and the inner circle must be contained in the outer circle. However, for convenience we shall also add that the origin coincides with the centre of the inner circle. As we saw in part I [35, Fig. 19], this representation is robust to distortion. Even if the circles are not concentric or the spokes are not equally spaced, the decoding produces a spiky polygon whose angles and edge lengths are quite varied, yet that still looks star-like. The lattice [45] of relationships between the subcategories is

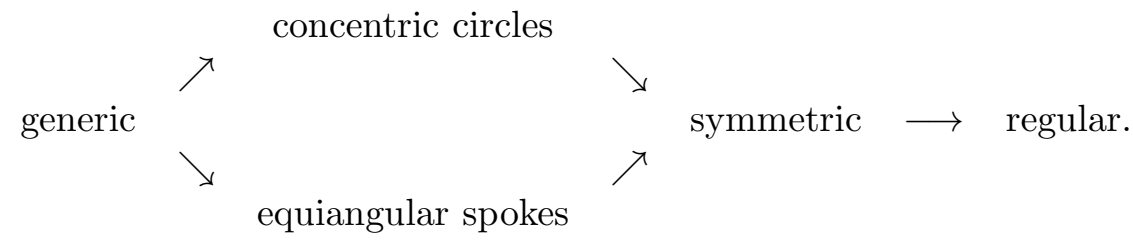

The generic star-like motifs with no special properties are on the left. The spacing of the spokes and the positioning of the circles are independent choices; when both are well controlled, the motif is symmetric. On the right, the mathematician's regular star with collinear lines is an exceptional case that occurs only when the ratio of the radii is just right - we know that this star belongs to a different category based on another construction.

The results of this thought experiment on generated categories give confidence that the features and relationships abstracted in the wheel representation are consistent with those used by perceptual and cognitive processes. They also provide another example in which spatial primitives based on qualitative properties like alignment perform better than those based on quantitative properties like lengths and angles.

\footnotetext{
${ }^{7}$ Bobick and Richards use this kind of parametrised algorithm as a generative representation to explore categorisation methods. They even use a turtle drawing a star polygon as a case study and note that closure is not a typical property of the category [12].
} 


\section{$7 \quad$ Size of representations}

How do we measure the size of a representation? This deceptively simple question does not have a straightforward answer, and the following observations illustrate some of the challenges. The size of a representation may depend on the choice of primitive objects and relationships used as building blocks in the representing space. A representation need not be a self-contained unit with a natural boundary; if it is dependent on a large archive of supplementary data and processes, how do we take their contribution into account? If the size of a representation is related to the entropy of the data it represents, we could compute a lower bound. An upper bound is more tricky. We can sustain arbitrarily large and complicated hierarchical representations (the layout of objects in a room, rooms in a building, buildings on a campus, streets in a town, and so on). However, there seems to be some kind of upper bound on representing detail at a single level. When the structure in the data becomes too complicated, the representation abstracts the gist or statistical features typical of a category rather than specifics of the given instance. Recall invokes a generative process that creates a random member of the category, not the original instance - the imagination will supply a different sample each time $[56,70]$. In this section we shall explore these issues.

Consider the following thought experiment. Suppose we have a set of primitive objects and relations that we can use to build representations of items of interest in our environment. We can assume that the number of independent primitives is finite and, to judge from the example of geometric patterns in the previous section, that the number is likely to be quite small. Let us measure the size of a representation by the total number, $N$, of primitives used in its construction. Then we can draw the following inferences.

- For any given $N$ there is a finite number of discrete configurations of $N$ elements.

- Hence, there is a small number of representations up to a given size.

- Hence, there is a small number of objects whose structure has a simple representation.

- Hence, objects that have structures we can fully comprehend are exceptional.

Although there are some further assumptions and generalisations that underlie this train of thought, it does not seem unreasonable.

The conclusion that comprehensible objects are exceptional does not mean that such objects are anomalies or rare in the environment, or that rare things have small representations. If the fundamental building blocks and relations are well adapted to the natural environment and the needs of the observer then we should expect objects that are common and important to have economical representations.

As we let $N$ increase and we enumerate larger and larger representations, we may start to repeat ourselves - that is, we may find different representations of the same object. This can arise for two reasons. The first is mechanical: the smaller representation can be obtained from the larger one by eliminating some redundancy. This is the compression process exploited by Chaitin and Schmidhuber. The second is semantic: the two representations express different interpretations or meanings. This cognitive ambiguity presents a choice between equally valid coherent structures (representing a star motif as a zig-zag polygon or a ring of kites for example), unlike the perceptual ambiguity illustrated in Figure 2, which is indeterminate and does not resolve into a stable structure at all. 
In ordinary conditions, the need to be able to act quickly means we cannot tolerate visual ambiguity or uncertainty. However, art dwells in an environment where it is safe for us to take time to explore various interpretations, and objects can have a mixed identity and play multiple roles. Some scholars have even argued that semantic ambiguity is an essential ingredient in art and plays an important role in determining aesthetic appreciation (see [84] for a recent survey). Ambiguity disrupts processing fluency, leading to a hard-to-process signal and the active participation of the viewer. The artwork presents a challenge, but it must also be comprehensible, otherwise we lose interest. Being able to resolve the data in multiple ways is not a barrier to comprehension - it means we remain engaged and can revisit familiar works. It is the experience of gaining insight rather than solving the puzzle that contributes to the appeal of ambiguous art [87].

The universal structures identified in layer 1 are based on a fixed set of primitive features that are derived from statistical properties of the natural environment and are expected to occur in the data, together with simple relationships such as proximity and local alignment. The higher-level structures discovered in layer 2 can be novel arrangements of unfamiliar elements. Even though we do not know exactly what we are looking for, spatial structure follows some general principles such as REPETITION and the PART-OF schemas, which help us find it.

- A composite structure has separate parts. Parts need not be localised self-contained units with sharp boundaries delineating one from another. Decomposition into parts is the result of grouping by strength of relationships: a part has a higher density of connections, and its constituents have more and stronger relationships between themselves than they do with elements outside. Although this seems indeterminate, it is analogous to grouping by proximity where there is no preset threshold for closeness?

- In a modular structure some of the parts are repeated. Reuse of standardised parts introduces redundancy into the representation of the structure, making it compressible. There are different kinds of repetition. In a geometric context, isometries (rotation, reflection, translation) and scaling are detectable by the visual system, but inflation rules are not (see $\S 12.5)$.

- In a hierarchical structure parts are assembled into larger units or, equivalently, parts can contain smaller parts.

The discussion above applies to any kind of representation. Let us now study a few examples to see how size varies in practice. The following representations are constructive and are presented as recipes. Taking this algorithmic approach means adopting a sequential symbolic language and, to produce economical representations, this coding language should support the features and structures we expect to encounter in the data. This includes general purpose control structures like subroutines and loops to capture the parts and repetition that are characteristic of higher-order structure.

For our examples, the coding language must capture the basic elements of geometry. At the beginning of part I we demonstrated how a complex Islamic pattern grew from simple ideas and various kinds of repetition: we divided a circle into 24 equal parts, taking

\footnotetext{
${ }^{8}$ Identifying parts and hierarchical levels is analogous to the mathematical problem of graph clustering (see [96] for a survey).
} 
two of its adjacent radii we constructed an equilateral zig-zag line by walking compasses alternately along them, the zig-zag was reflected, the resulting bilateral shape was repeated with 4-fold rotational symmetry, the resulting motif was combined with 10-pointed stars and some irregular fillers to form a square template, and the template was repeated by reflection in its sides. This is typical of geometric pattern constructions and illustrates the kinds of objects and operations often used: lines and circles, division into equal parts, grouping parts, and isometric transformations.

The coding language could be enriched with domain-specific primitives. We could extend the list of primitive objects to include shapes like kites and stars that occur frequently in Islamic patterns. We could add operations to construct a shape as the intersection, union, or difference of two given shapes, to find the bisectors of angles or line segments, to find perpendicular and parallel lines given a point and a line, and so on. We could even add the repetition rules of the basic symmetry types such as $p 4 m$. Even though using such a rich and specialised language may enable us to produce concise representations of patterns, we shall restrict ourselves to the basics.

We also need to establish how the coding language represents space. Is distance determined intrinsically from existing points or by an absolute unit of length? Is it measured relative to the current position or to a fixed origin? Is direction measured relative to the current direction or an extrinsic reference direction? Answers to these questions determine a frame of reference and a corresponding geometry: egocentric (e.g. turtle geometry), allocentric (synthetic geometry), or extrinsic (coordinate geometry).

The following examples all use the PostScript [1] page description language to draw the star motif of Figure 6(a). Although we shall only use a few basic elements, Postscript is a rich procedural programming language, having a broad range of control structures and data structures. Its most unfamiliar feature is the use of reverse Polish notation (arguments before operator). The representation encoded by the program is device independent, but some properties of any realisation such as resolution are determined by the display device. It does not matter if you do not follow the details of the code - the pros and cons of individual recipes will be explained.

Recipe 2 draws a piecewise linear path using a Cartesian coordinate system. This extrinsic frame of reference is determined by the page boundary and has an absolute unit of distance set at $1 / 72$ inch. By convention, the origin is positioned in the lower-left corner of the page or viewing window. In all the examples, the star motif is centred on the origin, so the either the figure or the viewing window needs to be translated for the complete figure to be visible. The recipe moves the cursor to a start position, lays a path with a sequence of straight lines, then closes the path by connecting the current point to the start point. The final command stroke then paints the path. This representation of the star as a 14-sided polygon does not reveal any of its structural properties - equilateral sides, symmetry, collinearity. It could be any 14-sided polygon.

The reliance on the coordinate system to convey metric information has two consequences. First, coordinates have an inherent graininess determined by the precision used for the values. If the required points cannot be expressed in rational fractions of a unit, their positions are inaccurate. Second, the intrinsic properties of the star used to compute the coordinates are hidden in an off-line process and the representation is just a record of the results.

Recipe 3 has an implicit framework of circles and spokes like the red lines in Figure 6(d). 


\begin{tabular}{rr}
\hline 0.000 & 50.000 moveto \\
9.655 & 7.699 lineto \\
48.746 & -11.126 lineto \\
5.358 & -11.126 lineto \\
-21.694 & -45.048 lineto \\
-12.039 & -2.748 lineto \\
-39.092 & 31.174 lineto \\
0.000 & 12.349 lineto \\
39.092 & 31.174 lineto \\
12.039 & -2.748 lineto \\
21.694 & -45.048 lineto \\
-5.358 & -11.126 lineto \\
-48.746 & -11.126 lineto \\
-9.655 & 7.699 lineto \\
closepath & \\
stroke
\end{tabular}

Recipe 2: Draw a piecewise linear path that closes up on itself.

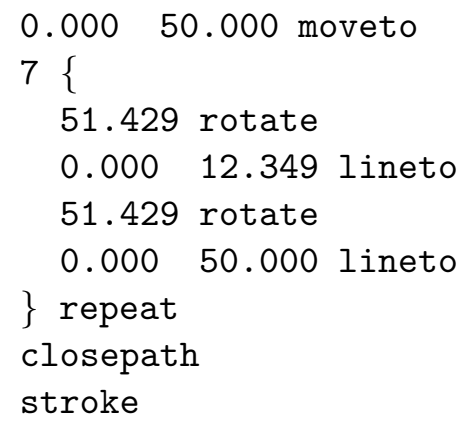

Recipe 3: Draw a zig-zag polygon using a wheel framework. 


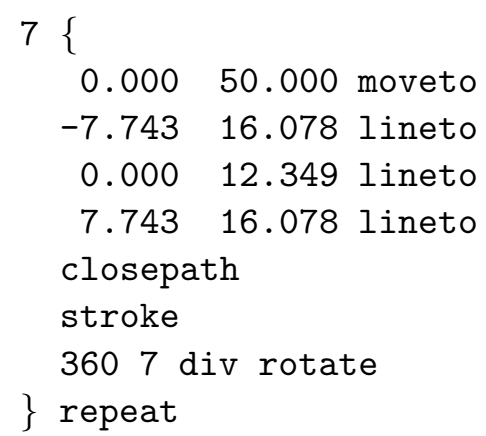

Recipe 4: Draw a 7-fold figure using seven copies of a 4-sided polygon.

First, it moves the cursor to an outer corner, then it draws lines, alternating the corners between the inner and outer circles, and rotating the figure by a constant angle as it progresses to move on to the next spoke. Finally, it closes the figure and paints the path. This representation clearly produces a zig-zag polygon. Without doing further computation, it is not obvious that the path has collinear lines, that the path crosses itself, or that this creates kites as a side-effect. The angle of rotation (approximately $513 / 7^{\circ}$ ) is hardwired; unless you recognise or verify that this is one-seventh of a full turn, it is not clear that the polygon has 7 -fold symmetry.

In Recipe 4 the moveto - lineto - ...-closepath - stroke sequence occurs inside the loop. This representation draws seven copies of a 4 -sided polygon. The rotation angle is now computed to the degree of precision available in the interpreter rather than coded as an approximation, so the result has 7 -fold symmetry. The polygon is a kite, and the star motif is represented as a symmetrical ring of kites. (Note that since the lines of the kite do not cross each other, we could use fill instead of stroke for an effect like Figure 6(b).) Because the mirror line of the kite lies on the $y$-axis, its symmetry is apparent in the coordinates. However, it is not clear that the complete figure is connected, or that the lines in one kite are continuations of lines in adjacent kites.

Recipe 5 is a revision of Recipe 4 . The kite has become an independent entity and has been coded in its own subroutine (like a chunk). This means the loop itself is much shorter. For efficiency, the calculation of the angle has also been moved outside the loop, storing the result in a local variable rather than redoing it each time. The code follows the convention that tokens in lower case are keywords in the language, and those in upper case are user-defined symbols.

In Recipe 6 the rules for drawing a star based on the representation in Figure 6(d) have been abstracted into a subroutine (or schema) with three parameters - the number of points and the radii of the inner and outer circles. To get a star with collinear lines, you have to supply radii in the correct ratio. The subroutine draws a single zig-zag polygon so it only works for an odd number of points. The first three lines are the mechanism for passing values into the subroutine (take them off the stack). Next it computes the rotation angle. The remainder of the code is just a parametrised version of Recipe 3. All the calculations are self-contained and do not rely on extrinsic properties such as the coordinate frame. The final line of the procedure has only four tokens and draws the star by calling the subroutine. 


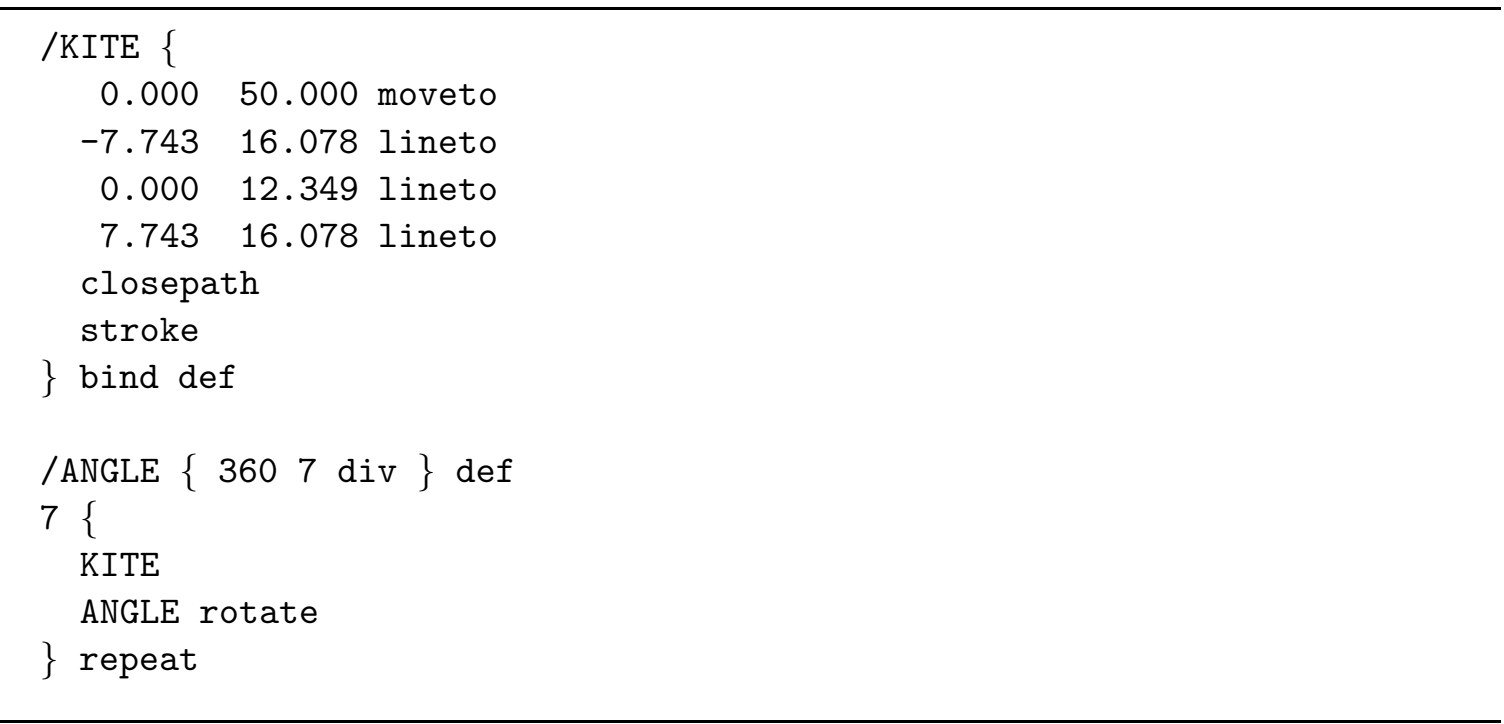

Recipe 5: Drawing the 4-sided kite is decoupled from the 7-fold symmetry.

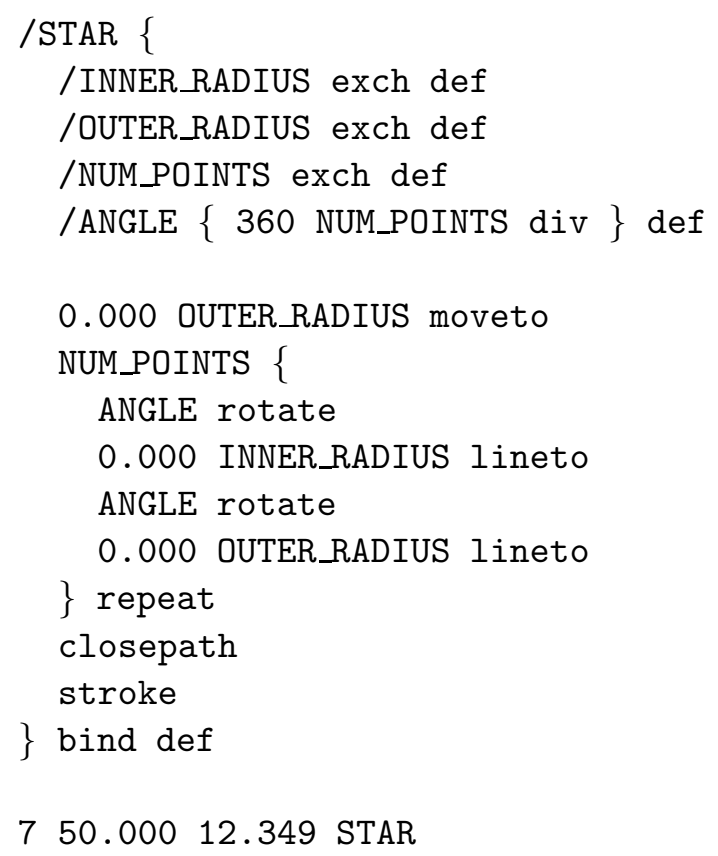

Recipe 6: A parametrised version of Recipe 3. 


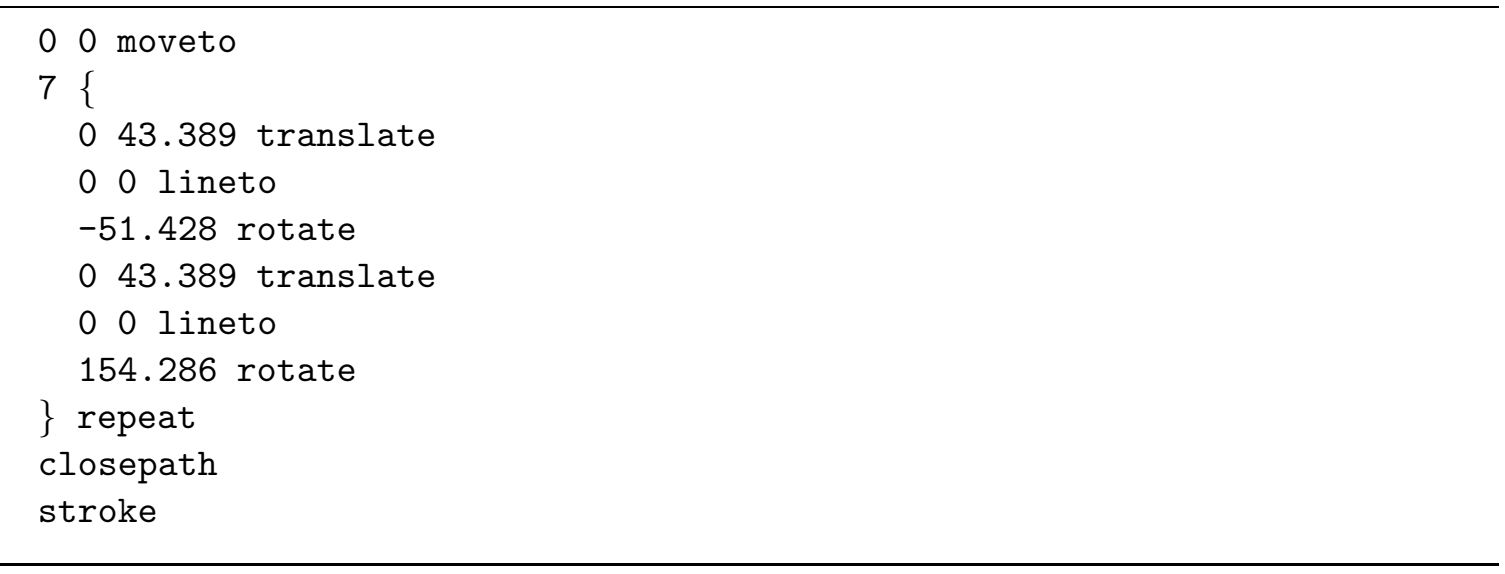

Recipe 7: A re-interpretation of Recipe 1: move the paper, not the pen.

\begin{tabular}{|l|ccccccc|}
\hline & Math. & Rec. 1 & Rec. 2 & Rec. 3 & Rec. 4 & Rec. 5 & Rec. 6 \\
\hline zig-zag & - & $\checkmark$ & - & $\checkmark$ & - & - & $\checkmark$ \\
polygon & - & - & $\checkmark$ & $\checkmark$ & - & - & $\checkmark$ \\
connected & - & $\checkmark$ & $\checkmark$ & $\checkmark$ & - & - & $\checkmark$ \\
collinear lines & $\checkmark$ & - & - & - & - & - & - \\
crossing lines & - & - & - & - & - & - & - \\
kites & - & - & - & - & $\checkmark$ & $\checkmark$ & - \\
symmetry & $\checkmark$ & - & - & - & $\checkmark$ & $\checkmark$ & $\checkmark$ \\
geometry & allo & ego & extr & allo & extr & extr & allo \\
size (tokens) & n/a & $25^{\dagger}$ & 44 & 19 & 22 & $19+14$ & $40+4$ \\
\hline
\end{tabular}

Table 1: Comparison of features belonging to representations of the star motif in Figure 6(a). $\dagger$ To make the counts comparable this value is for Recipe 7. 
We have presented eight different ways to represent the 7-pointed star motif in Figure 6(a): the mathematician's star, the craftsman's wheel, and six algorithmic coded recipes. How are we to choose between them? Table 1 evaluates the representations against some simple visual properties of the motif. The craftsman's wheel is not listed separately as it underlies Recipes 3 and 6 . A tick in a column indicates that it is immediately clear from the representation that the motif has the specified property. The properties in the rows are: is a zig-zag line, is a closed polygon, is connected in a single piece, has line segments that are collinear, has lines that cross, contains a kite as a submotif, has a centre of rotational symmetry. The geometry property indicates whether the representation uses an egocentric, allocentric, or extrinsic frame of reference. Although coordinates are used in most recipes, sometimes they express an absolute position (extrinsic), while at other times they give the distance from a centre (allocentric).

The final row gives an indication of the size of the representation by counting tokens in the PostScript codes. A token is a keyword, left or right brace, number, or named symbol. To make the comparison fair, the turtle code in Recipe 1 is recast as PostScript in Recipe 7 (think of it as moving the paper rather than the robot). The mathematician's representation is difficult to reproduce in PostScript. Representing a motif as the difference of geometric shapes only works properly for ideal mathematical figures composed of lines with no thickness. Implementing the process in a graphic image is problematic. Removing line segments from Figure 6(c) creates new corners between the remaining lines. In the graphic, when two lines with a common endpoint meet at an angle, the join needs a filler on the outer edge of the path to give a rounded or mitred finish to the corner. It is hard to produce this calculated result by drawing the star polygon, then erasing parts of it.

For Recipes 5 and 6 the size is shown as a sum of two contributions, corresponding to the subroutine and the main part of the code. In the first case, the use of a subroutine decouples the kite motif and the repetition aspects of the representation. The second case abstracts a generalised form of star. As mentioned earlier, the coding language could be enriched with domain-specific primitive objects such as kites and stars, and making use of such built-in features could shorten the code significantly.

Conversely, the coding language may be deficient in some respects, meaning that some structure in the data cannot be expressed. For example, PostScript has primitives that enable complex paths to be assembled from straight line segments, arcs of circles, and cubic Bézier curves, but there is no accessible operation to find the points of intersection of two paths. PostScript is designed to give a compact description of the appearance of an object on a page, not to perform all the computations required to construct the object. In particular, it does not simulate the Euclidean tools used in the constructions of synthetic geometry. If the object is constructed externally, the Cartesian coordinate system is an extrinsic reference frame that provides a PostScript mechanism to just 'print the answer'.

The coordinate format can be used even when it is possible to construct the object using PostScript primitives. Although the code in Recipe 2 is no shorter than the other recipes, for patterns that require a lot of computation, this kind of point-joining representation can lead to a substantial reduction in code size.

We do not pretend for a moment that cognitive representations are encoded like these examples. The recipes are presented more as a parable. They all contain sufficient information to reproduce the star motif to a degree of precision that the results cannot be distinguished by eye. Yet, although they all represent the same data, they do not abstract 
the same properties. None of the representations includes all the key visual features listed in Table 1. The line crossings, an obvious feature, do not play a role in any of the representations. If we use 'zig-zag' as a proxy for 'spiky' then Recipe 6 is the only representation that matches our earlier description of a star as a spiky, symmetric, polygon.

\section{Meaningful representations}

We have seen that the structure of even a simple star motif has different interpretations (zigzag polygon, ring of kites), and that these can be recorded in different kinds of representation (structural relationships, constructive recipe). It is not clear that any of them is a better representation than the others - there is no correct answer. This is partly because we have disregarded the function of the representation. Recall that a representation does not exist for its own sake, but to mediate between the input data and follow-on processes that can extract and use the information it encodes. Following Scheutz [98], we express this dependency as a 3 -term relation ' $R$ means $X$ to $P$ ' where $R$ is a representation or symbol, $X$ is a real or imaginary entity, and $P$ is a person or process. We say that $R$ is a meaningful representation, and note that it does not exist independently of $X$ or $P$.

We ignored $P$ in the examples of $\S 7$, but that does not imply that those representations are meaningless: they are lossless and completely regenerate the data so, in principle, they are universal representations - meaningful to any $P$. However, a representation can be selective, recording only the information salient to its downstream processes. The information can be filtered, abridged, or even reduced to a discriminating signature that triggers action but cannot be recalled.

Both of the links in the $X \longleftarrow R \longrightarrow P$ relation can be one-to-many or many-to-one relationships:

- An entity $X$ can be represented in multiple ways that reflect different interpretations, or focus on characteristics salient to different applications.

- A representation $R$ may abstract common properties from multiple objects, and thus represent a category or a metaphor.

- A representation may be general purpose - meaningful to many $P$.

- A process $P$ may bring information from multiple representations together to perform its function.

Representations can also be chained together as $X$ can be the output of a process, leading to a hierarchical network of processes that filter and assemble information. The bottom of this hierarchy interfaces with layer 1 of our mental model, where processes are passive (you get what you are given) and data-driven. At higher levels, and certainly at the interface with layer 3, we assume that processes are more active (you select what you need) and driven by the functions vital for survival and the conscious goals of the person. However, this network of representations is inaccessible and obscured in the opaque fog of layer 2 .

Consider the following example. Figure 7(a) shows a common pattern that contains $\{8 / 3\}$ star motifs, regular octagons, and examples of 5-pointed star shapes that do not conform to the wheel construction (they have a circumcircle but no inscribed circle). By exploiting global redundancy in the pattern, we can reduce it to the repeat unit shown enlarged in (b) - the pattern is formed from sixteen copies of this template repeated by 


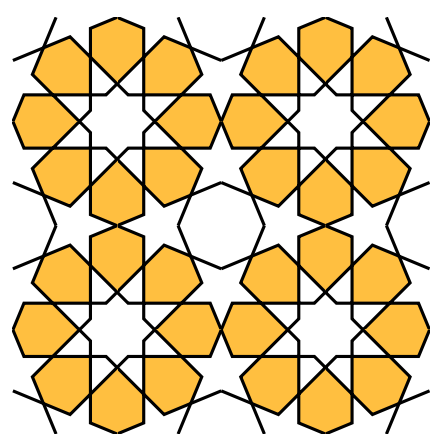

(a)

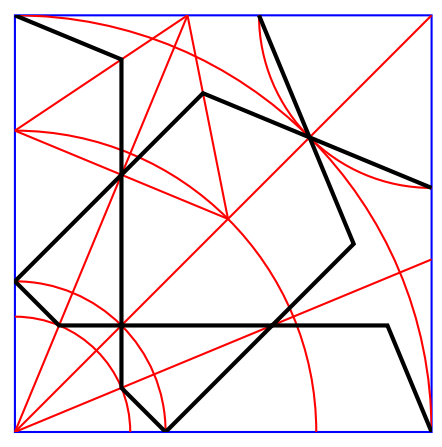

(b)

Figure 7: A common pattern and an annotated template that shows some of the relationships between salient points.

reflection, one of which is easily seen in the top-right corner of the pattern. The black lines in the template are the pattern, and the red lines capture local relationships in the form of linear and circular alignments between the key points (corners and crossings) in the pattern. The red circular arc that passes through the midpoint of the template does not meet any key points of the pattern, but it determines a line that does. The collection of red lines captures relationships that are sufficient to construct the pattern and are, therefore, a structural representation of the pattern. In fact, the red lines also illustrate a constructive representation because it is possible to find a sequence of simple steps from Euclidean geometry that produce this arrangement of lines. However, it requires reasoning and analysis to discover a sequence of logically dependent steps that result in such a procedure. Here, it is fairly straightforward (solution in the appendix), but this is not always the case. We shall return to this in $\S 10$.

This example is typical of templates found in medieval manuscripts that show pattern lines, and are annotated with construction lines. There is no explanatory text, just the pattern and key relationships, but there is sufficient information for a knowledgeable craftsman to recreate the whole pattern. This construction begins with the square boundary of the template and ends by finding the scale of the star - exactly the sequence that would be required to fit the design into its allocated space. It is a decorator's representation.

It is unlikely that this representation, expressed in low-level primitives, will help a typical viewer to make sense of the pattern. The more succinct and descriptive representation ' 8 -fold rosettes on a square grid' uses a richer language of high-level structures that has names for both the motif and the arrangement - the what and the where. A knowledgeable viewer with the relevant vocabulary and schemas could easily abstract these fundamental elements and produce this narrative that explains the pattern, and which is probably close to the artist's creative process that inspired the composition? Even though the description is so short, it contains enough information to enable an experienced person to reconstruct the pattern: the motif has a standard form, the absence of information to the contrary leads

\footnotetext{
${ }^{9}$ The distinction between the processes for creating and applying patterns is lost in many of the modern how-to books containing instructions for drawing Islamic geometric patterns. They do not teach the reader the rules of composition to create new works in the Islamic style, merely to follow point-joining recipes that reproduce traditional designs.
} 
one to assume a dense packing and standard alignment of the rosettes, and the basic rules of line extension produce the fillers to complete the design. Note, however, that someone who can deploy such ready-made schemas to deliver effortless comprehension with no deviation from the expected form will find the pattern pleasing but not interesting; puzzling over the sequence of steps in the decorator's template requires conscious engagement.

The decorator's template is a structural representation that shows how the fundamental parts (lines and corners) relate to each other. The associated construction is a lossless encoding, but does it make the pattern comprehensible? The short descriptive representation is expressed in terms of ready-made schemas. Like the last line of Recipe 6, it is highly compressed but hides the complexity by referring to a domain-specific library of known forms. The representation we first become aware of in layer 3 is more like the symbolic description, a holistic Gestalt that we have to consciously deconstruct to recover the smaller parts.

This is the difference between compression and comprehension. In the context of ' $R$ means $X$ to $P^{\prime}$ ', comprehension is associated with the relation $R \longrightarrow P$ whereas, when people discuss compression, they are usually concerned with encoding and hence the relation $R \longrightarrow X$. Compression is what you get at the interface of layers 1 and 2 ; it abstracts correlations and local features, and, while it does organise the data, it does not understand the bigger picture. Comprehension is what you get at the interface of layers 2 and 3; it provides a relatable coherent story that explains how the pieces fit together and makes sense of the data.

\section{Economical representations}

How do we measure the value of a representation? This is another simple question that does not have a straightforward answer. However, it is reasonable to assume that some kind of cost-benefit performance measure has improved as the visual system evolved, and that it is influenced by factors such as speed, accuracy, utility, robustness, use of resources, and so on.

One of the fundamental problems in vision is the everyday task of inferring a 3-dimensional environment from 2-dimensional data. One approach to resolve the inherent ambiguity is to invoke the parsimony hypothesis, a strategy for choosing between different explanations that make sense of data: do not make things more complicated than necessary and pick the simplest one. The choice takes various forms depending on the context: the theory with fewest assumptions, the model with fewest parameters, the shortest algorithm. Note that the strategy does not require optimisation - that is, do not enumerate all possible explanations to find the best, just choose from those presented. It also only applies when the options provide similar explanatory power, otherwise there is a trade off between simplicity and accuracy. For example, circular orbits are simpler (have fewer parameters), but elliptical orbits fit the data better.

The idea that evolution would favour parsimony in perception and cognition occurs early in the history of psychology and was proposed by Mach [74, 75]. It also underlies the Gestalt principles of grouping and perceptual organisation, which are rooted in the principle of Prägnanz: visual data will be perceived in the simplest form. Hochberg and his coauthors $[65,66]$ were the first to quantify a simplicity principle and demonstrate it empirically. They took ambiguous line drawings that can be perceived as 2- or 3-dimensional, and produced scores based on counts of features in the data such as line segments, straight lines 
(ignoring crossings), corners, crossings, and ratios of such quantities. This enabled them to compute which perceptual organisation of the data was specified by the smallest amount of information and predict which interpretation was preferred. After working with impossible figures, Hochberg later concluded that this approach was too simplistic [64].

Where Hochberg aggregated many simple measures, more recent applications of the minimum principle to cognition $[26,27,29,46,76,116]$ focus on a single metric - the size of the representation. This is not as one-dimensional as it may appear. For example, if evolution has produced a mechanism such that optimising performance results in small representations as a side-effect, then size can be used as a proxy for assessing other factors. In some contexts, size is related to probability so that more likely events are encoded as smaller representations $[4,25,93]$. Omitting details from a representation can improve its robustness: the model is less likely to overfit the data and hence be more resistant to noise. Smaller representations may also be faster to process. However, it is not obvious that smaller is always better. A representation is not just an encoding of data, but also a summary of our understanding of it. When viewed as an explanation, a representation with more internal connections is more coherent, and one with more supporting evidence in the form of connections to existing knowledge has a stronger justification. In this sense, larger representations may correspond to more plausible interpretations and hence be preferable [49]. In any case, we have already seen that the size of a representation is not well-defined: it depends not only on the data, but also on the choice of primitive objects and relationships used as building blocks, the richness of this language and how pertinent it is for the environment it is modelling, and whether the representation is self-contained or makes use of a shared archive of supplementary data and processes.

Furthermore, trying to capture performance in a single measurement has limitations. Parsimony is often equated with economy, but economy is about maximising return on investment across multiple variables, not just minimising resources. The cost of storage (size of a representation) is only one item on a balance sheet that should also include the cost of production, the value or significance of the information, and other factors. Representations need to be created (encoded), stored, and recalled (decoded) - operations that incur what might be called usage costs in terms of processing load (time and space) and storage costs (space). Ideally, it should be possible to compare and match representations without decoding them, but some formats are better suited to this than others: it is difficult to search through constructive representations looking for similarities, for example. Representations are constrained to work within the limitations of working memory and the chunking mechanism. Representations involved in detecting and avoiding danger need to deliver information that induces action quickly. These aspects of performance are usually ignored in the psychology literature, though good discussions can be found in $[2,62]$.

\section{Symbolic representations and information theory}

The underlying premise of cognitive psychology is that the brain is an information processing system. This approach took root in the 1950s and 60s when electronic computers provided a source of inspiration, and sometimes a metaphor, for mental processes. It was also influenced by studies of communication (the exchange of information using symbols), both in the grammars of natural languages and the mathematics of information theory [30]. Symbolic representation was part of this trend. Unlike features, which summarise subsets of the data, 
symbols are arbitrary tokens assigned to stand for features, abstract entities or operations, and they provide the framework for constructive representations.

The application of information theory to visual perception brought new definitions: regularity in visual data became equated with redundancy, and encoding (the creation of representations) was associated with compression. Leeuwenberg, one of the pioneers of this approach, developed coding schemes to represent data as a sequence of symbols [72] and applied his method to frieze patterns consisting of a single rectilinear figure (reminiscent of Greek key patterns). The encoding mechanism, analogous to turtle geometry, traces the path with an egocentric frame of reference and records advances and turns. The narrow scope of the data set means that the alphabet of symbols is small, generating an initial code is easy, and reducing the encoding to a canonical form is efficient (reduction applies three rewriting rules based on repetition, reflection, and recurrence of substrings). This method, known as structural information theory (SIT), has been developed further [117], but the examples still use serial data.

Algorithmic information theory (AIT), developed independently by Solomonoff, Kolmogorov and Chaitin, encodes data as an algorithm (sequential, symbolic, constructive representation) whose output recreates the given data. In its mathematical formulation [73], it studies algorithmic representations of binary strings, but Chater has also uses this way of thinking as a heuristic model of visual representations [26, 27]. He does not give worked examples to illustrate encoding, but notes that the coding language used must be able to express perceptually relevant features in the data set of interest and model their environment. The binary digits of $\pi$ are often used as an example of an apparently random string that can be generated by a small algorithm [6], revealing the counterintuitive result that it is compressible. Note that the algorithm is merely a means of reproduction and offers no insight into the meaning of $\pi$ - changing the coefficients in the generating function will produce a sequence with the same complexity but no mathematical significance. Moreover, finding such an algorithm requires a time-consuming, essentially empirical search. To be a model of perceptual encoding, we need to understand how such constructive representations can be created efficiently. SIT is clear in this respect but, as we saw with the decorator's representation in $\S 8$, constructive representations can be costly to produce.

SIT and AIT both employ algorithms as representations and use code size to select between different interpretations of visual data. The PostScript examples in $\S 7$ explored various aspects of algorithmic representations and also exposed some problems. We observed at the end of $\S 6$ that the turtle geometry encoding scheme used in SIT is based on absolute metric properties (distance and angle) that are not perceived reliably, and that the method does not abstract the correct category from the closed path of a star motif when the representation is parametrised. Using AIT as a high-level model leaves many practical details unresolved. Much of the mathematical theory concerns behaviour in the limit (arbitrarily long binary strings) and therefore applies to large data. Geometric patterns have a lot of redundancy and can be generated with short algorithms - compressing small strings is difficult $[39,50]$. Furthermore, the serial nature of algorithms does not capture the 2-dimensional structure of patterns very well, and more appropriate ways to quantify and represent the information in non-linear data are still being developed [3, 121, 122, 123]. 


\section{Discussion}

Geometric patterns do not have any of the attributes that we commonly associate with European Fine Art. They do not depict anything - even the ones with interlaced ribbons do not pretend to show a 3-dimensional woven object [113]. They have no title. Usually we do not know who created them. We know little about the art-historical context the artist worked in or the artist's intention. The viewer may recognise some of the polygonal shapes, and even know names for some common ones, but the shapes carry no meaning. Geometry is cold - it has no emotive content and does not evoke memories to make you feel anything. The traditional patterns or motifs may once have had cultural associations or symbolic value, but this is now lost and certainly unknown to a modern viewer who is unfamiliar with Islamic philosophy and culture. Grabar, one of the leading art historians and scholars of Islamic art, addressed the problem of meaning ${ }^{10}$ and concluded that geometry is just a game [53]. It is partly the absence of these confounding variables that makes geometric patterns good stimuli for psychology experiments. Despite the lack of associations, we can still engage with a pattern for its own sake.

A consistent thread through the writing on interest is that it lies on a spectrum that runs from familiar-predictable-boring at one end to arbitrary-chaotic-confusing at the other. Interesting things fall somewhere between these extremes - they are neither too simple nor too complicated to understand. This psychological instance of the Goldilocks principle was noted by Wundt [120], the founder of experimental psychology, in the 1870s, and again by Berlyne [9] a century later in his experimental aesthetics. Furthermore, the position on the spectrum varies as we become more experienced and our level of understanding increases: previously seen stimuli become easier to process and therefore less interesting. We like novelty, but not so much that we cannot make sense of it. This is expressed in Silvia's appraisal model as dysfluent yet comprehensible.

Many applications of psychology use dual-process models. Their basic format is a division of mental processes into two contrasting classes: intuitive or logical, autonomous or controlled, passive or active, fast or slow. It is common for many such characteristics to be aggregated, even though it is unclear that the boundaries are aligned. (Indeed, dualprocess theories have been criticised for clustering sundry dichotomies without any evidence $[43,82]$.$) The model presented in Figure 1$ uses two unaligned dichotomies to create three layers ${ }^{11}$ objective-automatic, subjective-automatic, and subjective-conscious. These correspond to the divisions in the two bottom rows of the table. The first layer is mostly driven by the stimulus and detects low-level features that are expected to occur in the environment; the second layer learns from experience, recognising familiar structures that have been stored as chunks or schemas, and discovering relationships and organising features to build new structures. The first two layers are inaccessible to conscious thought; layer 3

\footnotetext{
${ }^{10}$ Grabar concluded that patterns are ornament - superficial and decorative. Blair and Broom also consider the purpose of patterns is 'to stir appreciation and pleasure in the eye of the beholder and encourage him or her to linger, think and delight' [11]. Others believe patterns operate on a deeper level as art and, therefore, carry meaning if we know how to find it. For example, geometry may communicate Neo-Platonic ideals or express mystical symbolism in the Sufi tradition. See the discussion in [119] for a brief overview of the debate.

${ }^{11}$ Stanovich also has a 3-layer model [108], but whereas we have divided System 1 to produce our layers 1 and 2, he divides System 2 and calls his three layers autonomous, algorithmic (strategies), and reflective (beliefs, goals).
} 
concerns things we know we know. Layer 1 performs general purpose processing that is the same in everyone; last two layers depend on the experience and goals of the viewer. Spreading out mental processes in this way exposes the scope of layer 2, where most of our exploration has taken place. It is not so explicit in the literature, but it is important in our discussion.

Many relationships in visual data are determined by correlation. Fine-grained shortrange correlation and discontinuities in homogeneity define lines and regions. Correlations in the orientations of lines are evidence of alignment and good continuation. Coarse-grained correlation leads to properties like texture. Correlating attributes (grouping by similarity or common motion, for example) starts to identify parts. Long-range correlation in space or time finds repeated configurations and common context. Correlations in multimodal data acquired when we interact with a physical object allow us to see that some parts correspond to natural structures and that correlations may have environmental causes. Finding correlations has two consequences. One is volume reduction as a collection of related things can be summarised by a single entity (points become a line). The second is that stored correlations lead to expectations, and the ensuing surprise when an addition, omission or change in the environment produces an anomaly.

We have spent a lot of time exploring the properties of representations. The general concept has a tripartite form where the representation mediates between the represented entity and an agent or consumer who benefits from the information. Although much has been written about mental representations (see for example [40, 48, 86, 90, 92, 95, 101, 109]), we do not know what they are, how they function, how they handle different levels of abstraction, whether they are distributed and blended with one another or isolated, and so on. Nor do we have direct experience of the representation itself - it is encapsulated and inaccessible. Nevertheless, this mythical device plays a useful role in shaping the discussion. It is a tool that helps us to examine the interaction between the viewer and the pattern, and to infer which parts of the data are salient and which are ignored or go unnoticed.

Without getting too philosophical about what is actually being represented, we note there are things a representation is not doing. A representation is not a lossless encoding of the raw signal detected by the sensor; the data is filtered, selected, and correlated to produce low-level features before it reaches layer 2, where representations are maintained. Therefore, the raw ingredients of representations are lines, corners, crossings and so on. Furthermore, a representation is driven by the needs of the consumer ${ }^{12}$ not the content of the data. This means that irrelevant or distracting details can be eliminated to reduce the processing load, so long as the representation holds the information required to generate an appropriate response to the current situation. It can be a course-grained summary that distorts reality, so long as the significant relationships are maintained.

Which properties of geometric patterns are recognised as important by the visual system? First note that visual processing does not depend on the accurate measurement of lengths and angles, but relies heavily on relative positions, comparative measures, and qualitative attributes and relationships, which are simpler and more robust. This includes properties like connectedness, straightness, parallelism, collinearity, and more general forms

\footnotetext{
${ }^{12}$ Constructing representations of patterns does not have immediate survival value, so it is unclear what consumer needs are being met by doing so. However, all cultures produce patterns, so they must exercise our visual processing systems in pleasing ways and provide puzzles that satisfy our drive to make sense of incoming data [28].
} 
of alignment. This approach is consistent with a synthetic geometry built on betweenness of points, and equivalence relations for parallel lines, lengths, and angles — no absolute measures, just comparison. It is distinguished from Euclidean geometry by adding the pragmatic axiom that any length or angle can be divided into any number of equal parts.

In part I I defined regularities as those features or structures in the data that are processed fluently. In that case, we focussed on perceptual fluency (processes in layer 1 of our model) and noted that experimentally determined regularities seem to coincide with non-accidental relationships in the data (edges, straightness, alignment, centres of local symmetry, and so on). Here in part II I shall retain this definition and consider the higherorder regularities determined by the cognitive fluency of the processes in layer 2. Some structural regularities are consequences of general purpose mechanisms that circumvent the limited capacity of working memory. Universal primitive spatial schemas abstract simple structures that recur in our everyday interaction with the environment: REPETITION is the defining property of patterns, CENTRE plus REPETITION leads to rotational symmetry, GROUP and PATH could underlie some of the Gestalt principles of perceptual organisation. The PART-OF schema is so important that it is singled out as a mechanism in its own right: chunking identifies assemblies of parts, repeated or interchangeable parts (modularity), and nested parts (hierarchy). We also develop personal domain-specific schemas such as STAR.

Processing fluency, and hence regularities, are determined by the interaction between the viewer and the pattern. Perceptual regularities depend on properties of the pattern and properties of the visual system. Cognitive regularities are more subjective and also depend on the degree of openness, the spatial processing style, and the experience of the viewer. Detecting regularities is part of what makes a pattern comprehensible: identifying parts, how they fit together, and how they work together as a whole. However, understanding how all the elements relate to one another does not mean you can produce a copy the pattern. The catalogue of detected relationships may not contain sufficient information to determine unique proportions or positions of all the elements in the pattern. It may also be inconsistent so that the relationships cannot all be satisfied simultaneously. It is not until the viewer tries to reproduce the pattern that it becomes apparent that anything is amiss. This is also a problem for the artist, who has to convert an idea into a viable composition. It is unclear how this lack of shared understanding affects the relationship between the viewer and the artist - for a typical viewer, making sense of a pattern cannot involve recovering the underlying idea, recognising the challenges it entails, and appreciating the skill and ingenuity of the artist in solving them. When the artist successfully prioritises relationships to be in sympathy with the visual system, the resulting artwork appears natural and effortless.

Over time, artists learned to control the geometric medium and work with and around its constraints. In the next section we shall see that there is a clear trend in the structure of patterns: artists became much better at maintaining perceptual regularities like alignments, and patterns became more complex and more organised in accordance with cognitively detectable regularities — more modular and more hierarchical.

\section{The evolution of Islamic geometric patterns}

In the following illustrations of the evolution of Islamic patterns, we are not only tracing their development over time. There is a Darwinian aspect to the composition of patterns: a collection of geometric patterns exhibits variation in motifs, their arrangements, orienta- 
tions, connections, and so on; new patterns can be created by modifying existing patterns or combining known elements in new ways; the successful results provide the raw material for the next cycle of experiments. The best patterns are widely used, the best motifs become part of the standard vocabulary for the style, and other ideas persist for a while until they evolve into or are displaced by something better, possibly something completely new.

While many cultures perpetuate traditional patterns unchanged for generations, in the medieval period, Islamic patterns underwent continual innovation. The evolutionary process is driven by competition and selection, and the motivation for this study was explore whether psychology can help explain what 'successful' means in this context: what properties of patterns influence the choices made by an artist? In two papers we have applied our current understanding of the physiological, perceptual and cognitive aspects of human vision to predict which features make geometric patterns pleasing or interesting.

Evolution operates with episodes of gradual development punctuated by disruptive events that transform the environment. In our case, such events include contact with other cultures through trade or invasion, the introduction of new methods or materials, the discovery of new technology such as coloured glaze, or changing the criteria for success by taking the cost of production into account as well as visual impact. We do not have space for a detailed history of Islamic patterns so we shall concentrate on a few substantial shifts in direction that may be loosely described as the introductions of stars, colour, modular design, hierarchy, and modern mathematics.

\subsection{Stars}

Recall from $\S 6$ the star motifs that mathematicians denote $\{n / p\}$ : they are constructed by placing $n$ points equally spaced around a circle and connecting all pairs of points $p$ steps apart with straight lines. Star motifs of this form predate Islamic patterns. The following examples are found in Classical mosaics [8, pp. 88-91]: $\{6 / 2\}$ (compound of two triangles), $\{8 / 2\}$ (two squares) and $\{9 / 3\}$ (three triangles). The stars in early Islamic patterns are

also based on these simple forms, but inner line segments are removed to leave only the outer contour or the characteristic ring of kites. Figure 8 shows patterns formed from dense arrangements of stars based on $\{6 / 2\},\{8 / 2\},\{8 / 3\},\{10 / 3\}$ and $\{12 / 3\}$. These few examples already illustrate that the same star can be arranged in different ways, and the same layout can be used with different stars. The circumcircles of the stars are packed together, and the stars are aligned so that their spikes meet where the circles are tangent. This means that the pattern lines continue from one star to another without a break or deviation. The voids between the circles are filled in the same manner: the lines of each star are extended outside its circumcircle until they meet lines from other stars. Note that in pattern (d) this line extension process fortuitously creates small kites that are congruent to those in the stars.

One consequence of the Crystallographic Restriction is that global centres of 5 -fold rotation cannot occur in periodic patterns. Anyone designing repeating patterns will soon discover this empirically. Patterns containing 5-pointed and 10-pointed stars hint that they can evade this constraint, even though such stars will be local rather than global centres of symmetry. Besides having 10-fold motifs, Figure 8(d) is arranged on a rhombic grid, which helps to add interest - patterns arranged on square and triangular grids have higherorder symmetry, which imposes more constraints on the designer and makes the resulting 


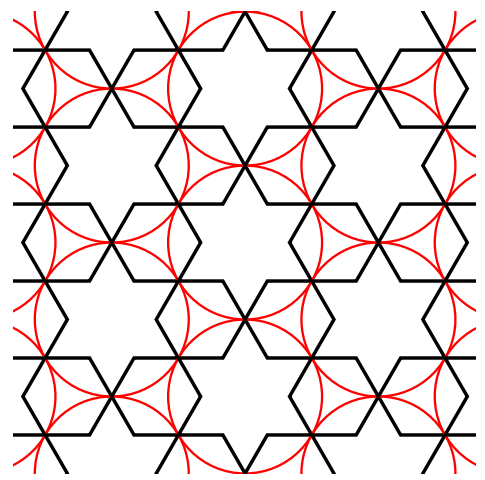

(a) $\{6 / 2\}$ on triangular grid

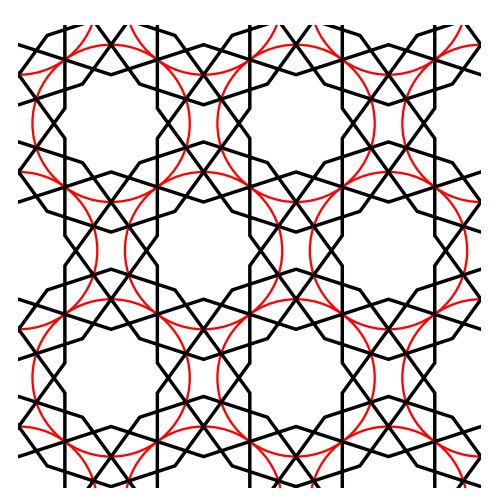

(d) $\{10 / 3\}$ on rhombic grid

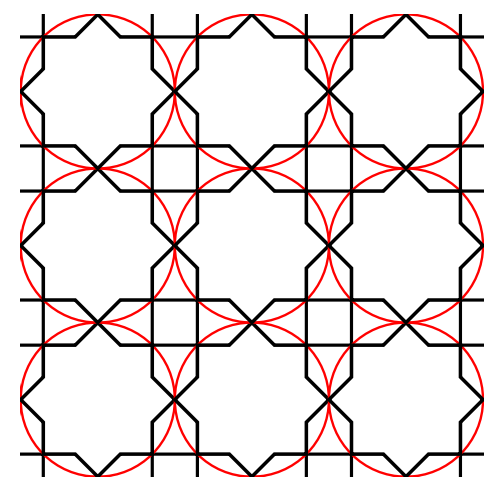

(b) $\{8 / 2\}$ on square grid

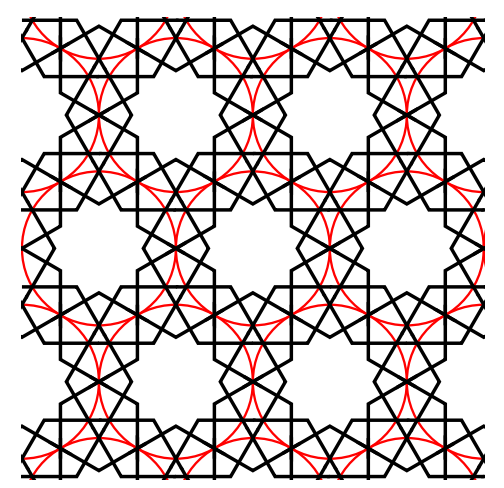

(e) $\{12 / 3\}$ on triangular grid

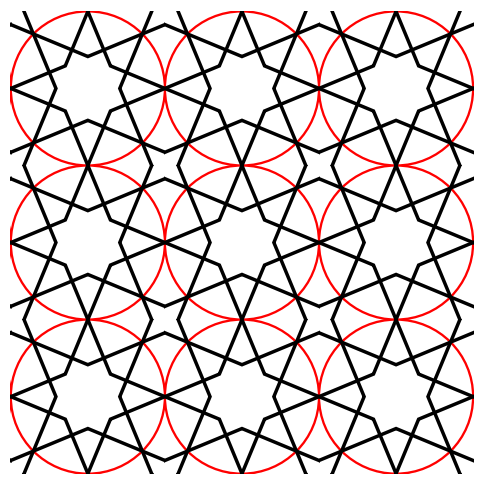

(c) $\{8 / 3\}$ on square grid

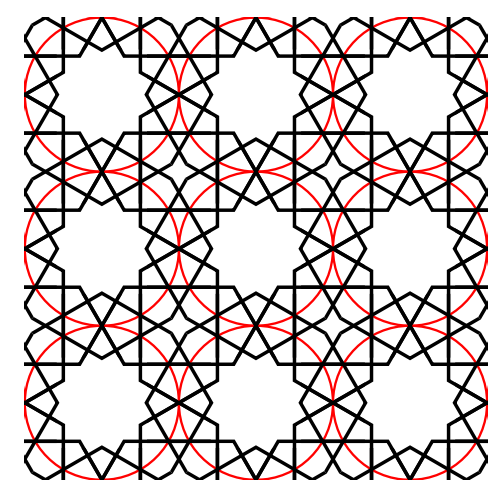

(f) $\{12 / 3\}$ on square grid

Figure 8: Dense arrangements of regular stars on standard grids. 
pattern more predictable for the viewer. This pattern is widespread and quite common, and is elaborated in many variations. The introduction and frequent use of 5-fold and 10fold motifs is a significant innovation in Islamic patterns and is one of their distinguishing features.

So far we have seen some straightforward arrangements of a single kind of star. Later patterns use combinations of different stars. The simplest and earliest one combines $\{5 / 2\}$ (the pentagram) and $\{10 / 4\}$ - see [35, Fig. 22]. These star motifs have compatible geometry: ten small stars fit naturally around a large star in such a way that everything aligns and connects perfectly. Extending this constellation to a periodic pattern requires minor adjustments to some of the small stars but is straightforward. Figure 9(a) and (b) shows two patterns having 12-pointed stars arranged on triangular and square grids. The primary stars are based on $\{12 / 5\}$, but the 5-pointed stars are not symmetric. The relative sizes and arrangement of the motifs are determined by packing the circumcircles of the stars.

Some traditional patterns mix two, three or even four kinds of large stars, motifs which are distributed so that they are both connected and separated by 5-pointed stars. In most cases, motifs based on $\{n / p\}$ stars are too rigid for this type of composition and a more general approach like the wheel construction is required to ensure good continuation of the pattern lines where stars meet. In part I we saw two examples where the primary motifs are 9-pointed and 12-pointed stars [35, Fig. 5], and 11-pointed and 14-pointed stars [35, Fig. 25]. In terms of construction and visual appearance, these two patterns are very similar, but mathematically they are very different: in the first the constituents are compatible with each other and with the underlying grid that governs the layout, while the intuitive interpretation of the second as an arrangement of symmetrical stars violates the laws of geometry.

Figure 9(c) shows another illicit example, this time mixing 11-pointed and 13-pointed stars. Because a small misalignment between elements is easily noticed, the construction shown here prioritises alignment over symmetry: the spokes of the wheels are collinear with spokes defining neighbouring stars, but this means the spokes cannot be equally spaced around a wheel and hence the resulting stars are slightly irregular. The framework defined by the spokes is shown in blue on the left of the figure. The red circles reveal the imperfect geometry. The small circles are inscribed in some of the triangles in the blue framework and, at first glance, they seem to play a similar role to the circles in Figure 9(a) and (b). However, they differ in size and do not pack together precisely, either overlapping or leaving gaps. This technique exploits the fact that the STAR schema used by the visual system summarises data as idealised forms, so the deception is not noticeable without conscious detailed analysis.

The development of this family of patterns with dense arrangements of stars displays a typical progression of increasing complexity: a single kind of star, adding 5-pointed satellite stars to help negotiate awkward transitions, multiple kinds of large stars with compatible geometry, and finally breaking free of mathematical constraints to produce illicit combinations of conflicting geometries.

\subsection{Colour}

The introduction of colour as a major element in Islamic patterns followed the rediscovery of techniques for glazing ceramics. At first, lead-based clear green glaze was used to insert small highlights. The more vibrant tin-based opaque glazes were initially applied to strips 


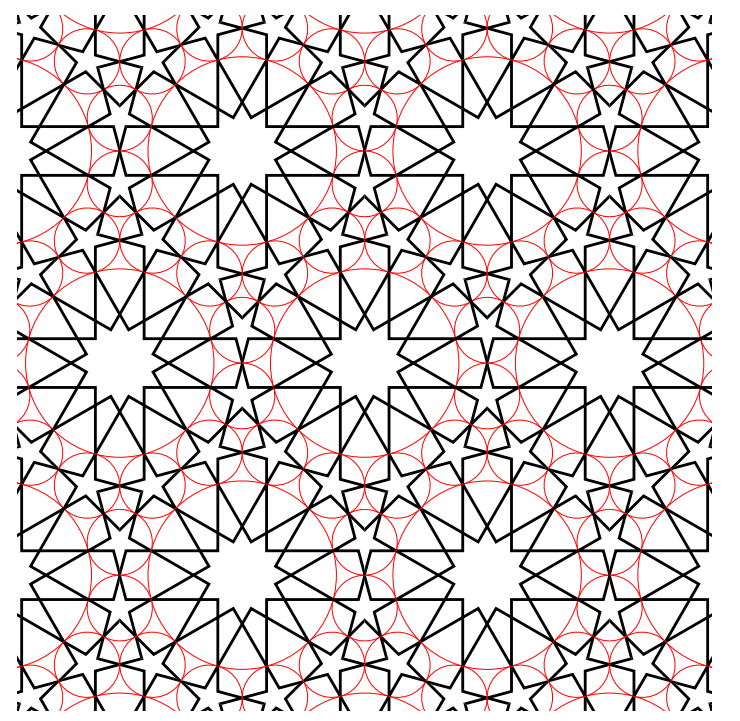

(a)

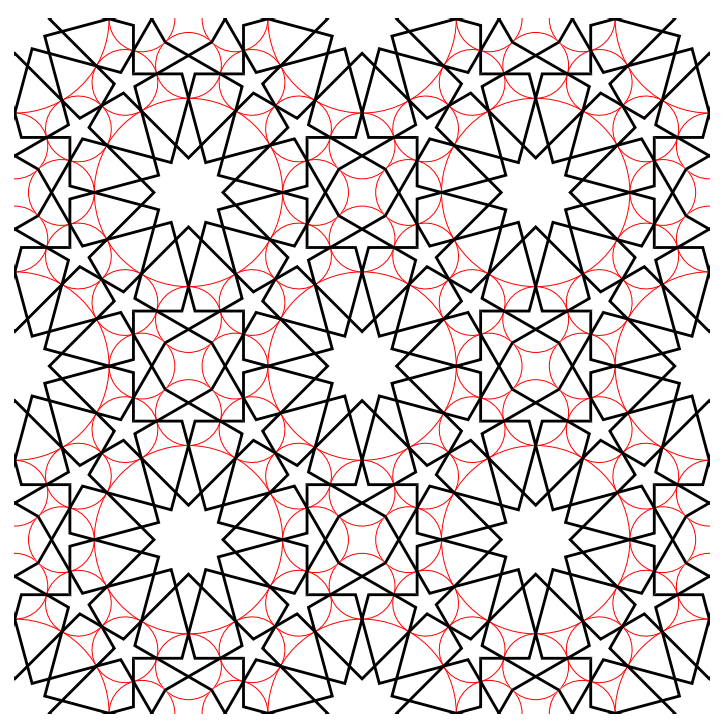

(b)

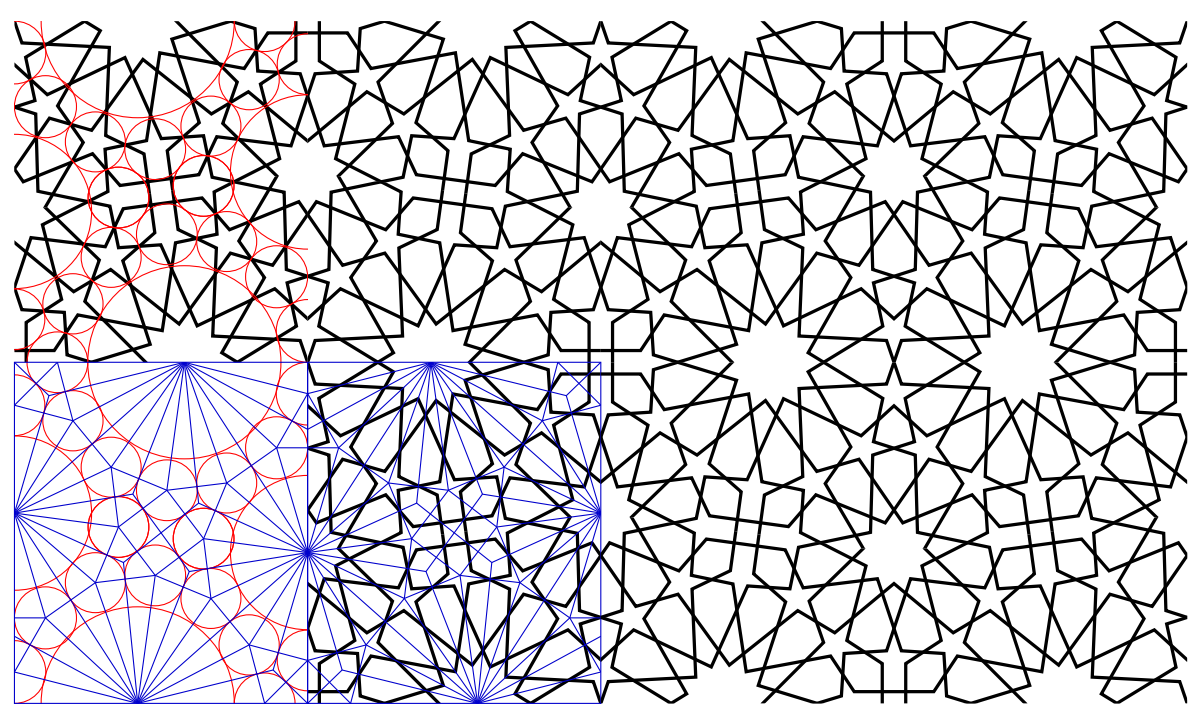

(c)

Figure 9: Constellation patterns. 


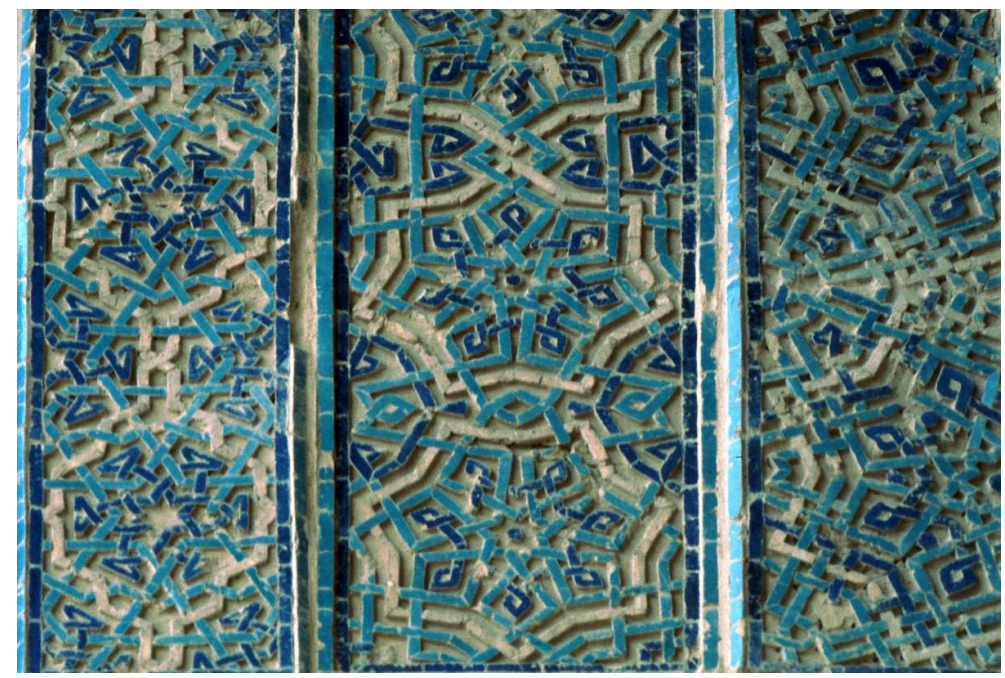

Figure 10: Three panels with 6-fold, 7-fold and 8-fold motifs made of coloured strips, mosque at Varamin, Iran.

of terracotta and used for inscriptions and linear patterns like those in Figure 10. With the mass production of glazed tiles, it became possible to have continuous coloured surfaces: shapes were cut from monochromatic tiles and assembled as a mosaic with colour contrast defining and accentuating the pattern. However, large panels of intricate cut-tile mosaics are time-consuming and expensive to produce, and eventually geometrical patterns were displaced by square tiles painted with floral arabesque.

The colour of a glaze depends on its composition (metal oxides), and the atmosphere (oxidation or reduction) and temperature in the kiln. As understanding of the process developed, the vivid turquoise of the first glaze was supplemented by a range of colours in more subdued tones: white, black, dark blue and light blue, green, yellow, tan and brown, and a very dark purple sometimes called aubergine. The consistency of the chemistry gives a remarkably uniform colour palette across a large geographical area, and yields another recognisable characteristic of Islamic style.

Colour adds another channel for distinguishing multiple parts. Consider the two versions of a star pattern shown in Figure 11. The one on the left is the packing of 12-pointed stars that we saw in Figure 8(e); on the right, some of the lines have been coloured blue. The addition of colour changes our interpretation and we see a fusion of two complementary patterns that have the same weight and scale, and fit together such that the corners and crossings of one lie in the open spaces of the other, and vice versa. The blue pattern is the same as Figure $8(\mathrm{a})$ (rotated by $90^{\circ}$ ) and the black pattern can be seen as a composition of overlapping hexagons - both were used independently as traditional patterns in their own right. Grouping by colour is a strong perceptual effect that is hard to override: in the monochrome pattern the large hexagons are still quite prominent, but the 6-pointed stars are hard to isolate.

The example in Figure 11 may have been discovered through serendipity, simply by adding colour to an existing pattern. However, other complementary patterns were constructed deliberately by weaving strands of different colours through familiar patterns. Figure 12 shows two examples: in (a) the blue pattern is traditional and is overlaid by a 


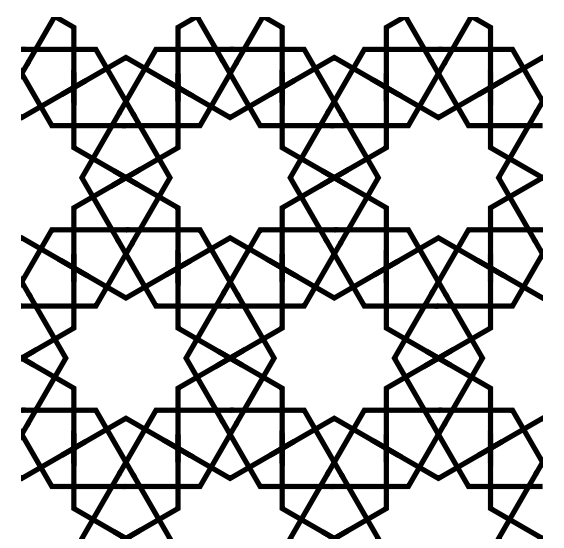

(a)

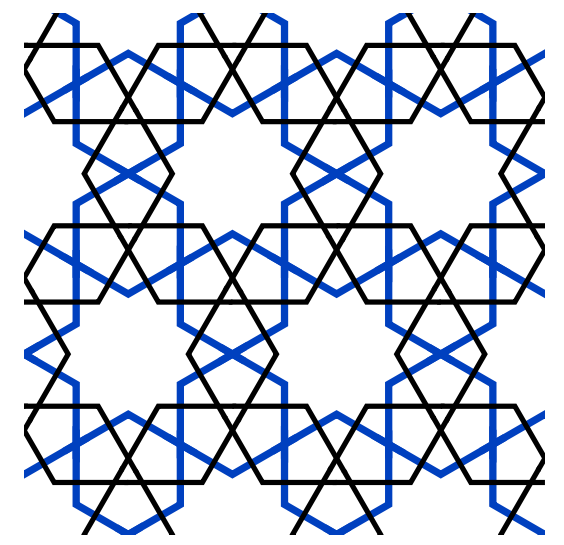

(b)

Figure 11: The impact of colour on design.

complementary interlaced pattern that is also based on traditional motifs; in (b) the packing of 6-pointed stars is used on two different scales - a simple example of hierarchical structure that we shall return to below.

\subsection{Modular design}

A modular design system is a small but versatile set of basic elements (modules) that can be assembled to produce a large variety of structures. Modules are often grouped into units that are then repeated, but free-form arrangements that grow more organically are also possible. Modular design requires less technical skill to produce successful results as the process naturally creates a balanced distribution of related elements.

Bricks, the primary building material in Iran and Central Asia, provide an obvious example of modular construction, and they were also used for decoration [68]. At first, this was achieved by varying bonding patterns, or offsetting to produce relief patterns that cast harsh shadows in the strong sun. Glazed and unglazed bricks were mixed to imitate weaving patterns - a technique known as banna' $i$. In later buildings, the structural and decorative functions of bricks were decoupled and a decorative revetement was attached to the walls, which allowed greater variety in the shape and placement of the modules, even employing specially moulded or carved bricks. But brick patterns are inherently limited by the rectangular form of the medium, and very few of them contain star motifs.

Figure 13 shows two patterns that contain stars embedded in a large matrix of other shapes. In each case, the matrix has structure - the same few shapes recur in the same few local configurations. This is true within each pattern individually, but notice that the same shapes and configurations are also shared between the patterns. These are indicators of modular design. Figure 13(b) shows a Hankin diagram (see part I): the overlaid red lines bisect the crossings and form a segmentation of the pattern. Here we shall disregard the debate about whether the modules are the polygons bounded by the red lines, or are the compartments bounded by the lines of the pattern. In either case, you find a small number of different shapes meeting in a small number of local configurations.

The panel in Figure 13(a) is from the Topkapı Palace in Istanbul, and is decorated with pieces of ivory, tortoiseshell and mother-of-pearl. There are many similar panels in the 


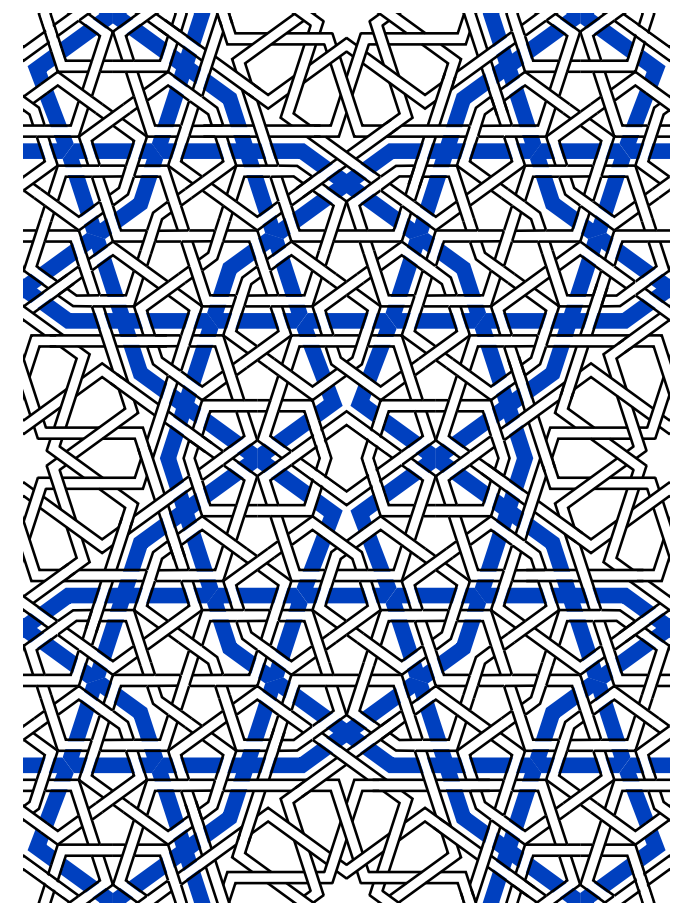

(a)

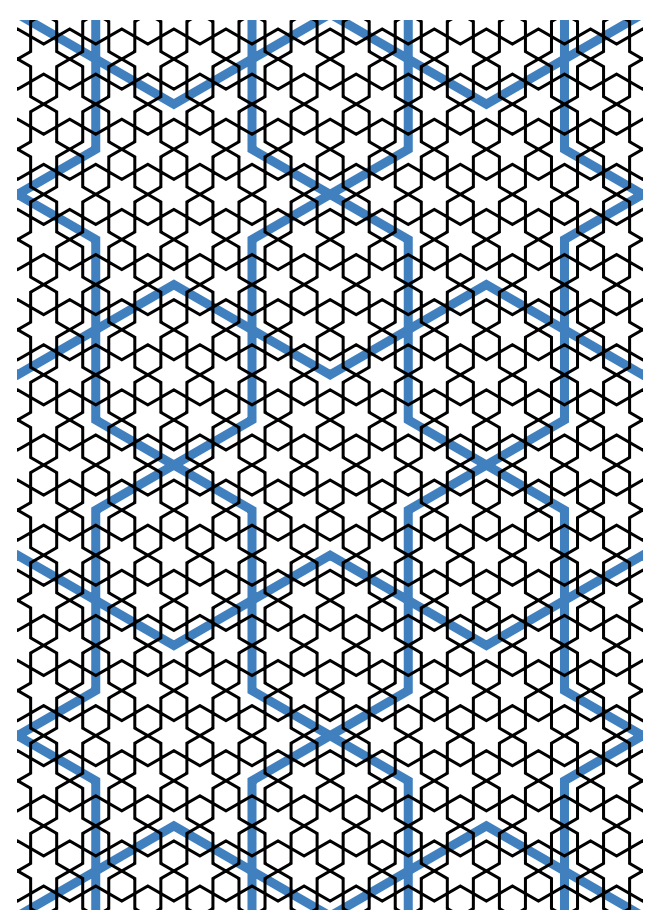

(b)

Figure 12: Designs by the author: composite structures with colour defining the different parts. (c) P. R. Cromwell.

palace, particularly in the kiosks and the harem, and many more examples can be found on the doors, shutters and furniture in the city's mosques. Even though there are many different patterns, they all feel familiar. They clearly belong to the same family and this homogeneity brings unity and cohesion to the decorative scheme. However, there is so much redundancy that they become formulaic and rather boring. They all share the same local properties and any discriminating features are on a large scale. Unless the patterns are near enough for direct comparison, they seem interchangeable - 'seen one, seen them all'.

Modular patterns can be made more interesting by occasionally breaking the rules to disrupt the viewer's expectations. Introducing such anomalies (what in crystallography would be called defects) can be accomplished in various ways:

- insert a new module into the system

- substitute modules from a different but related system (for example a $\{10 / 4\}$ star into a modular system based on the $\{10 / 3\}$ star) [32, Fig. 8]

- arrange the modules in a way that produces slight gaps or dislocations [36, Fig. 10]

- allow the configuration of modules to extend beyond the boundary of the repeat unit and crop those that overhang; modules that are not compatible with the boundary will create incongruous shapes in the pattern when the template is reflected (but the lines will join up) [32, Figs. 7, 11]. 


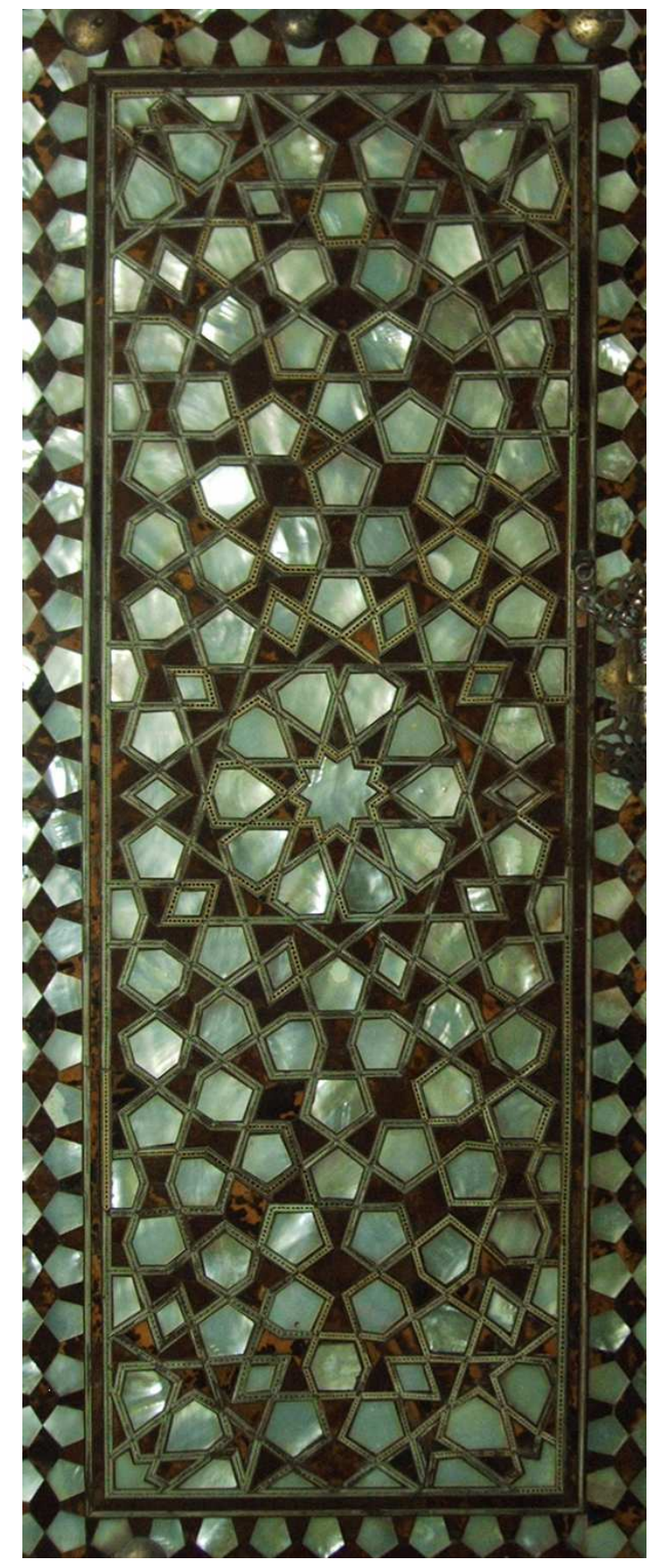

(a)

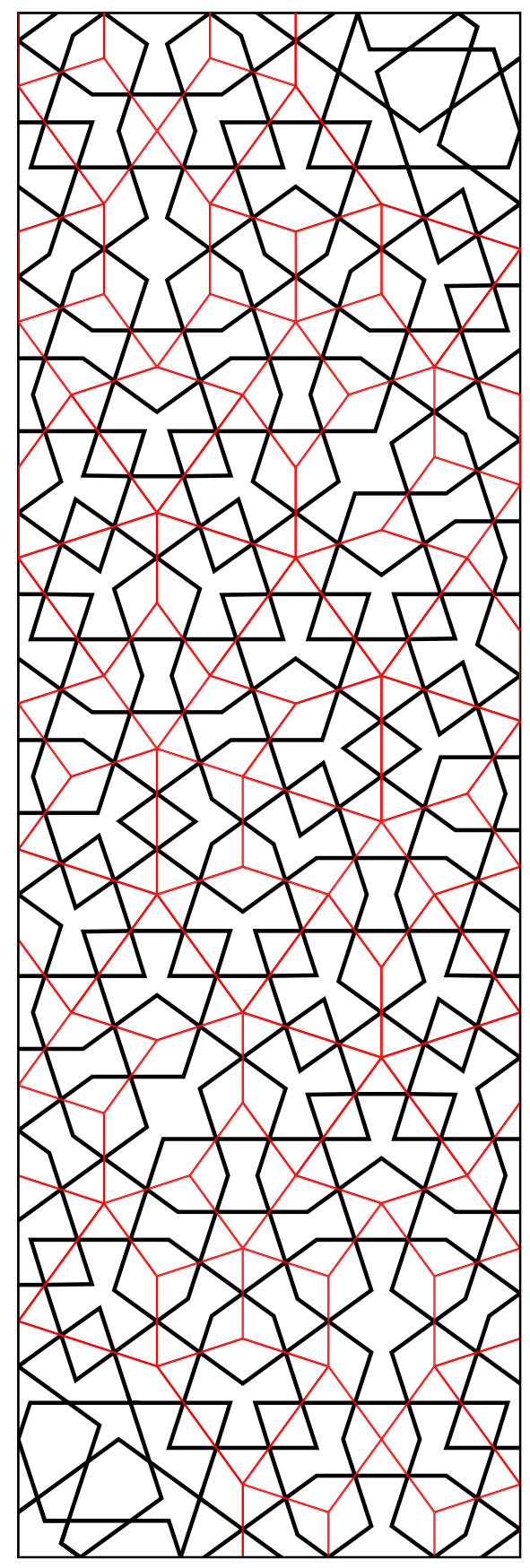

(b)

Figure 13: Two modular designs from doors in Istanbul: (a) is from the Topkapr Palace and (b) is a Hankin diagram of another design in the same family. 


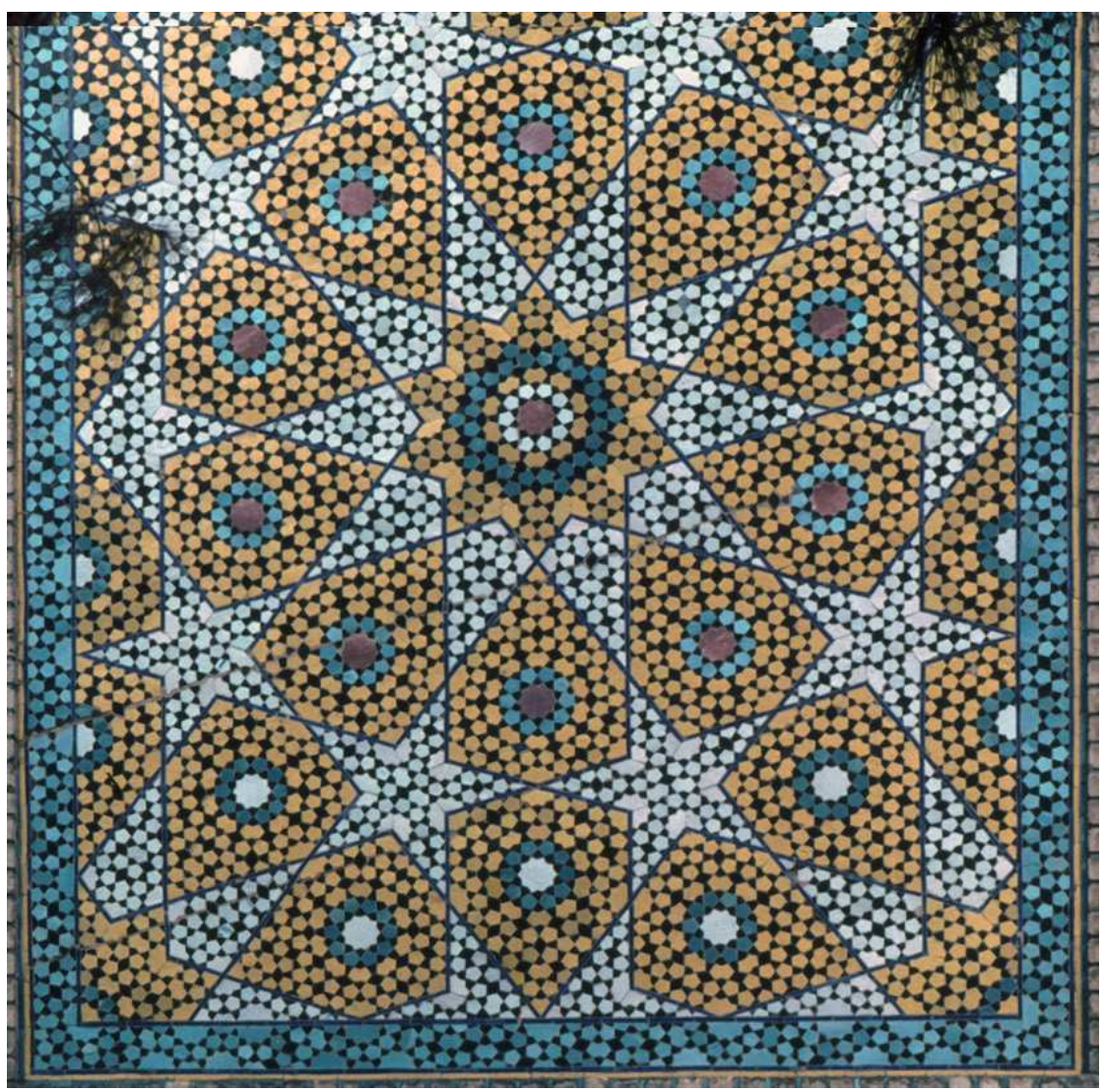

Figure 14: Hierarchical design from the Chahar Bagh Madrasa, Isfahan, Iran.

\subsection{Hierarchy}

Hierarchical structure has parts within parts. Hierarchy in ornament is often produced by the progressive filling of background areas with ever finer detail. Geometric patterns naturally provide a framework of compartments that can be furnished with smaller scale designs such as arabesques or calligraphy (as seen in Figure 3). Applying the geometric canon on the smaller scale imposes additional constraints: in Islamic ornament the filler is not merely a motif that occupies some of the space, but a pattern that should be coherent with its frame.

Figure 14 shows a late example of a hierarchical design. The large-scale pattern is displayed in white and gold and is a common, relatively simple star pattern that is easy to recognise. (It is also outlined for emphasis, but the colour difference is the discriminating feature noticeable from a distance.) The small-scale pattern is displayed in black on a multicoloured pale ground. If the ground were uniform and other indicators of the largescale pattern were removed, the small-scale structure would still be a valid, if somewhat busy, pattern in its own right. The two patterns are not independent parts juxtaposed in competition with each other, but are carefully coordinated so that the different elements support, highlight and complement one another. For example, the crossings and corners of the large-scale pattern are situated at the centres of stars in the small-scale pattern - these key points of the large pattern are in the largest open spaces available where interference 
from details of the small pattern is minimised. Colour and modular design are key to creating this kind of hierarchical Islamic pattern: the flexibility and control required to produce a pattern that fills a prescribed area is most easily achieved by using a modular system for the small-scale pattern [34].

The design is intricate and its execution as a mosaic lacks precision: the tesserae are cut by hand and are variable in shape and goodness of fit. The two patterns are based on different motifs (the large-scale on $\{10 / 4\}$ and the small-scale on $\{10 / 3\}$ ), but they have compatible geometry and the design does have a flawless archetype. The irregularities introduced by the craftsmen are regarded as noise in the data and will be ignored in the representation produced by our visual system. This strategy, which under normal conditions adds robustness to visual processing, also enables the artist to mislead the viewer. Some hierarchical designs apparently show constructions that are mathematically inconsistent [36]: the geometry of a modular system used for the small-scale pattern is incompatible with the shape of the area it is being used to fill. However, the method of manufacture makes it easy to disguise the deception.

\subsection{Modern designs}

Hierarchical design in various forms $[13,31]$ marked the high point of the classical tradition of Islamic geometric patterns. Like the modular design that made it possible, this genre, too, became formulaic. It was also expensive to produce, and fashion turned away from geometric patterns to floral ornament.

In non-Islamic ornament, hierarchy is sometimes produced by a recursive procedure that generates ever smaller levels of detail. The important thing is that the output of the process is the same kind of object as the input so the process can be iterated. Starting with a simple form like a line or a triangle, repeated subdivision or augmentation creates a finite fractal-like motif.

An analogous idea can be applied to create Islamic patterns. It requires a modular design system where modules can be assembled to make larger copies of themselves. A pattern is generated by taking a small patch of modules, subdividing each of the modules into smaller ones, then enlarging the result so that the small modules become the same size as the originals. By iterating this procedure, known as inflation, the pattern can cover any given area. While this sounds straightforward, it is difficult to find modular systems for which inflation generates something other than periodic patterns. For example, an inflation rule can be derived from Figure 12(b), but this pattern can be generated by simpler means.

In the 1970s Penrose discovered small modular systems that support inflation and produce repetitive patterns that are not periodic [59, Ch.10]. Although the same local configurations recur throughout the pattern, each one has a unique global context. Makovicky discovered more quasiperiodic tilings after studying Islamic patterns [77], and several people have used the Penrose tilings as the basis for new Islamic star patterns [14, 21, 32]. The example shown in Figure 15 is a hierarchical pattern where the large-scale pattern is derived from Penrose matching rules and the small-scale pattern is built from a traditional Islamic modular system (the same as that in Figure 14). To me, at least, the results are not entirely satisfying. There is clearly redundancy in the pattern, but the global organising principle remains elusive. We cannot work out how to extend the pattern beyond the piece shown. Although 'spot the flaw' experiments show that humans can identify a range of structural 


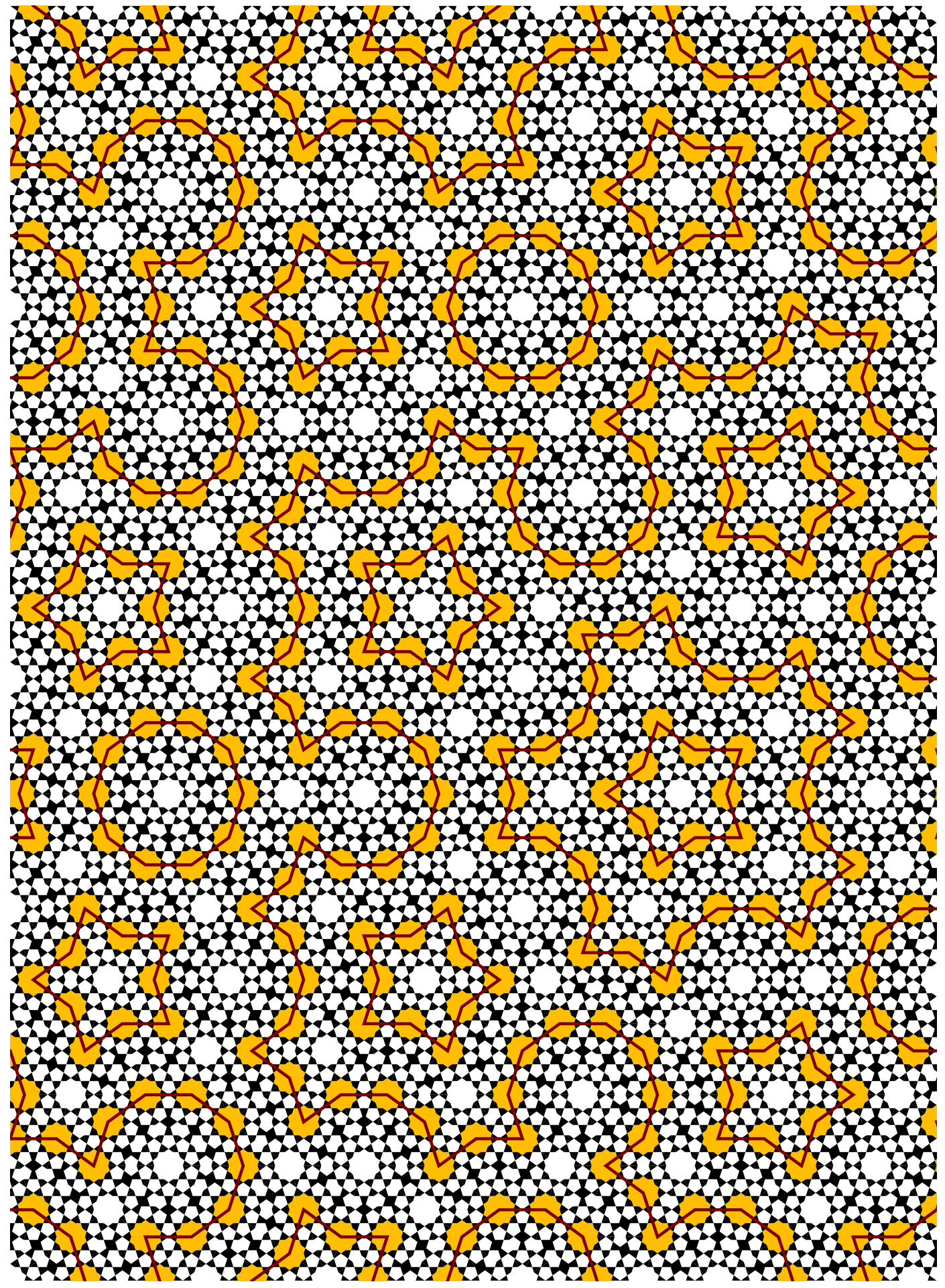

Figure 15: Design by the author based on a quasiperiodic Penrose tiling. (c) P. R. Cromwell. 
violations in rule-based patterns [118], we do not notice when Penrose tiles are assembled incorrectly. Trying to find evidence of quasiperiodicity in traditional Islamic patterns has been a cause of confusion and controversy [33]. The apparent complexity in Figure 15 is generated by six iterations of a small recursive PostScript program - very little information is required. However, inflation is a mathematical process that does not correspond to a physical one found in the natural world, and the visual system has not evolved to recognise it.

The final example illustrates one of the most surprising fusions I have seen in recent years, although the viewer needs some knowledge of cultural conventions to recognise and appreciate it. Jean-Marc Castéra is a designer with a lot of experience in the traditional arts of Morocco [21]. Following a visit to Iran in 2015, he produced new work by applying the techniques of the west to patterns from the east [22]. Figure 16 follows in this spirit. The large-scale pattern, highlighted in gold, is a standard Persian pattern (the same as the blue pattern in Figure 12(a)). The large-scale pattern is defined by colouring some of the small-scale compartments. In order to create these coloured channels, the stars must be aligned dent-to-dent (a western convention), whereas in the eastern tradition stars are mostly placed spike-to-spike. There are two sources of surprise here. The first comes from the incongruity of conflicting labels (eastern pattern, western treatment). The second comes from the fact that no-one had done this before. Medieval Persian craftsmen had both the large-scale pattern and the modular system used for the small-scale pattern, yet the conventions of the Persian style prevented them from seeing this possibility.

\section{Concluding remarks}

We set out to understand why Islamic geometric patterns give us pleasure, why they interest us sufficiently that we will spend time puzzling over them, and why some patterns do this better than others. This is not a simple exercise deploying existing knowledge and readymade solutions; in fact, patterns make a good test case to enrich our understanding of vision. Islamic patterns are novel stimuli for psychological study: more complicated and intricate than the 2-dimensional figures typically used in experiments, yet still highly structured with a limited range of variables. ${ }^{13}$

Throughout the study we have taken a holistic system approach and considered properties of both the stimulus and the viewer, and particularly the interactions and interdependencies between the two. This includes the innate processing capabilities, preferences and personality type of the viewer, plus properties and routines learned or assimilated through experience with the physical, social, and cultural environments. The focus on interaction was extended to the definition of regularities (features and structures in the stimulus processed fluently by the viewer), instead of regarding them solely as properties of the pattern expressed as symmetry transformations or compressibility. We also identified low-level and high-level structures with separate mechanisms in different parts of the processing chain, and divided visual processes into two major stages: universal and stimulus-driven followed by subjective and consumer-driven. Here a consumer is anyone who engages with the pattern, both the artist who creates it and a viewer who encounters the finished product.

\footnotetext{
${ }^{13}$ Although one can argue that the artists who created the patterns were experimenting on the visual system, the patterns were not contrived to systematically explore one variable at a time.
} 


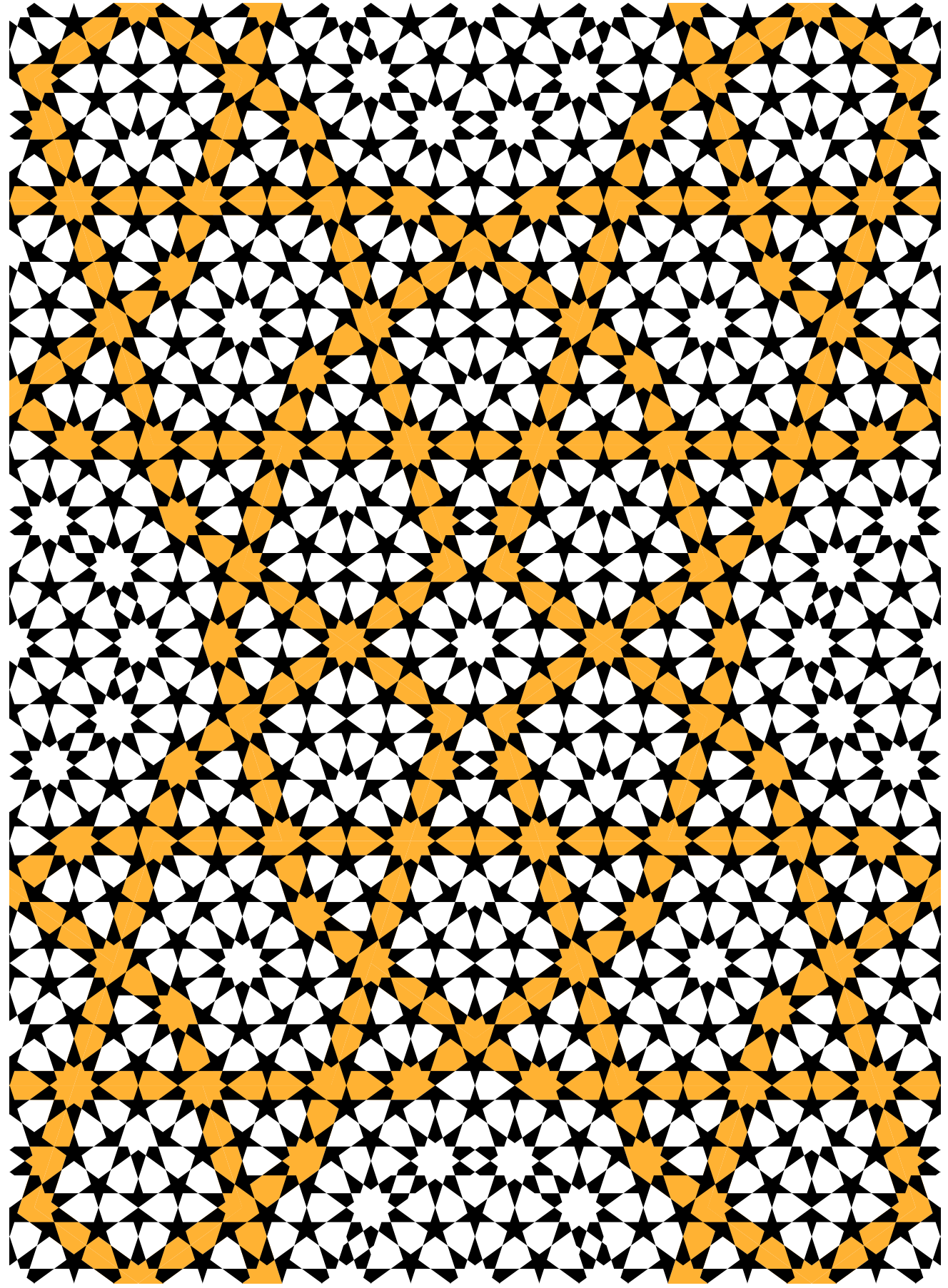

Figure 16: Design by the author: an eastern pattern with western treatment. (c) P. R. Cromwell. 
One outcome is that we have understood the psychological foundations of geometry as a visual science based on comparitive and qualitative properties rather than as an applied science concerned with absolute measures of distance, angles and area, or as a pure mathematical science concerned with consistency, accuracy and verification by logical argument. Some questions remain. In particular, we have ignored colour, which plays a significant role in the appreciation of later Islamic artworks executed in cut-tiles.

In $\S 7$ we mentioned in passing that semantic ambiguity, being able to support multiple interpretations, is a distinguishing characteristic of art. There are many papers that work towards a scientific theory of art or aesthetics, but this is not one of them. We have avoided the awkward questions of the philosophers and art historians, questions such as 'is it art?', 'is it beautiful?', 'why was it made?', and 'what does it say?'. We have focused more on 'how does it work?' in the sense of what effects it has on the viewer and what makes one pattern more pleasing or more interesting than another — we have begun to answer the Gestalt question 'is it good?'.

This research received no specific grant from any funding agency in the public, commercial, or not-for-profit sectors.

\section{Figure sources}

Figure 13(a) is by the author.

The other photographs are from David Wade's archive at http://www . patterninislamicart.com/.

and are used with permission. Figure 3 shows photographs EGY 1222, TUR 0409 and TUR 0826. Figure 10 is IRA 2929. Figure 14 is IRA 1116.

\section{References}

[1] Adobe Systems Inc., PostScript Language Reference Manual, third edition, AddisonWesley, 1999.

[2] A. Aksentijevic and K. Gibson, 'Psychological complexity and the cost of information processing', Theory and Psychology 22 no. 5 (2012) 572-590.

[3] Y. A. Andrienko, N. V. Brilliantov and J. Kurths, 'Complexity of two-dimensional patterns', European Physical J. B 15 (2000) 539-546.

[4] F. Attneave, 'Some informational aspects of visual perception', Psychological Review 61 no. 3 (1954) 183-193.

[5] A. D. Baddeley and G. Hitch, 'Working memory', The Psychology of Learning and Motivation, ed. G. H. Bower, Academic Press, 1974, pp. 47-89.

[6] D. H. Bailey, J. M. Borwein, P. B. Borwein and S. Plouffe, 'The quest for pi', Math. Intelligencer 19 no. 1 (1997) 50-57. 
[7] L. Ball, E. Threadgold, J. E. Marsh and B. T. Christensen, 'The effects of stimulus complexity and conceptual fluency on aesthetic judgments of abstract art: evidence for a default-interventionist account', Metaphor and Symbol 33 no. 3 (2018) 235-252.

[8] C. Balmelle and M.-P. Raynaud, Le Décor Géometrique de la Mosaïque Romaine: vol 2 Répertoire Graphique et Descriptif des Décors Céntres, Picard, 2002.

[9] D. E. Berlyne, Aesthetics and Psychobiology, Appleton-Century-Crofts, 1971.

[10] C. Bier, 'Geometry and the interpretation of meaning: two monuments in Iran', Proc. Bridges: Mathematical Connections in Art, Music, and Science, (Towson, 2002), ed. R. Sarhangi, 2002, pp. 67-78.

[11] S. S. Blair and J. M. Bloom, 'Cosmophilia and its critics: an overview of Islamic ornament', Beiträge zur Islamischen Kunst und Archäologie 3 (2012) 39-54.

[12] A. Bobick and W. Richards, Classifying Objects from Visual Information, A.I. Memo 879, A.I. Lab, M.I.T., 1986.

[13] J. Bonner, 'Three traditions of self-similarity in fourteenth and fifteenth century Islamic geometric ornament', Proc. ISAMA/Bridges: Mathematical Connections in Art, Music and Science, (Granada, 2003), eds. R. Sarhangi and N. Friedman, 2003, pp.1-12.

[14] J. Bonner, Islamic Geometric Patterns, Springer, 2017.

[15] J. Bourgoin, Les Éléments de l'Art Arabe: Le Trait des Entrelacs, Firmin-Didot, 1879. Plates reprinted in Arabic Geometric Pattern and Design, Dover Publications, 1973.

[16] V. F. Bruce, P. R. Green, M. A. Georgeson, Visual Perception: Physiology, Psychology and Ecology, Psychology Press, Taylor and Francis, 2003.

[17] N. Burgess, 'Spatial cognition and the brain', Annals New York Academy Sciences 1124 (2008) 77-97.

[18] D. T. Campbell, 'Variation and selective retention in socio-cultural evolution', Social Change in Developing Areas: A Reinterpretation of Evolutionary Theory, eds. H. R. Barringer, G. I. Blanksten and R. W. Mack, Schenkman, 1965, pp. 19-49.

[19] B. A. Cartwright and T. S. Collett, 'Landmark learning in bees: experiments and models', J. Comparative Physiology A 151 (1983) 521-543.

[20] B. A. Cartwright and T. S. Collett, 'Landmark maps for honeybees', Biological Cybernetics 57 (1987) 85-93.

[21] J.-M. Castéra, Arabesques: Art Décoratif au Maroc, ACR Edition, 1996.

[22] J.-M. Castéra, 'Persian Variations', Nexus Network J. 18 (2016) 223-274.

[23] J. C. Castro-Alonso and P. Jansen, 'Sex differences in visuospatial processing', Visuospatial Processing for Education in Health and Natural Sciences, ed. J. C. CastroAlonso, Springer Nature Switzerland AG, 2019, pp. 81-110. 
[24] G. Chaitin, 'The limits of reason', Scientific American 294 no. 3 (2006) 74-81.

[25] N. Chater, 'Reconciling simplicity and likelihood principles in perceptual organization', Psychological Review 103 no. 3 (1996) 566-581.

[26] N. Chater, 'The search for simplicity: a fundamental cognitive principle?', Quarterly J. Experimental Psychology 52A (1999) 273-302.

[27] N. Chater, 'A minimum description length principle for perception', Advances in Minimum Description Length - Theory and Applications, eds. P. D. Grünwald, I. J. Myung and M. A. Pitt, MIT Press, 2005, pp. 385-409.

[28] N. Chater and G. Loewenstein, 'The under-appreciated drive for sense-making', J. Economic Behavior and Organization 126 (2016) 137-154.

[29] N. Chater and P. Vitányi. 'Simplicity: a unifying principle in cognitive science?', Trends in Cognitive Sciences 7 no. 1 (2003) 19-22.

[30] T. M. Cover and J. A. Thomas, Elements of Information Theory, second edition, WileyInterscience, 2006.

[31] P. R. Cromwell, 'Analysis of a multilayered geometric pattern from the Friday Mosque in Yazd', J. Math. and the Arts 6 (2012) 159-168.

[32] P. R. Cromwell, 'Islamic geometric designs from the Topkapı Scroll II: a modular design system', J. Math. and the Arts 4 (2010) 119-136.

[33] P. R. Cromwell, 'Cognitive bias and claims of quasiperiodicity in traditional Islamic patterns', Math. Intelligencer 37 no 4 (2015) 30-44.

[34] P. R. Cromwell, 'Modularity and hierarchy in Persian geometric ornament', Nexus Network J. 18 (2016) 7-54.

[35] P. R. Cromwell, 'Looking at Islamic patterns I: the perception of order', Preprint 2021.

[36] P. R. Cromwell and E. Beltrami, 'The whirling kites of Isfahan: geometric variations on a theme', Math. Intelligencer 33 no 3 (2011) 84-93.

[37] J. E. Cutting, 'Perception and information', Annual Review of Psychology 38 (1987) $61-90$.

[38] S. Dehaene, V. Izard, P. Pica and E. Spelke, 'Core knowledge of geometry in an Amazonian indigene group', Science 311 (20 Jan 2006) 381-384.

[39] J.-P. Delahaye and H. Zenil, 'Numerical evaluation of the complexity of short strings: a glance into the innermost structure of algorithmic randomness', Applied Mathematics and Computation 219 (2012) 63-77.

[40] S. Edelman and S. Duvdevani-Bar, 'Similarity, connectionism, and the problem of representation in vision', Neural Computation 9 no. 4 (1997) 701-720.

[41] D. E. Egan and H. J. Schwartz, 'Chunking in recall of symbolic drawings', Memory and Cognition 7 no. 2 (1979) 149-158. 
[42] P. C. Ellsworth and K. R. Scherer, 'Appraisal processes in emotion', Handbook of Affective Sciences, eds. R. J. Davidson, K. R. Scherer and H. H. Goldsmith, Oxford Univ. Press, 2003, pp. 572-595.

[43] J. St. B. T. Evans and K. E. Stanovich, 'Dual-process theories of higher cognition: advancing the debate', Perspectives on Psychological Science 8 no. 3 (2013) 223-241.

[44] M. W. Eysenck and M. Keane, Cognitive Psychology: A Student's Handbook, fourth edition, Psychology Press, 2000.

[45] J. Feldman, 'The structure of perceptual categories', J. Mathematical Psychology 41 (1997) 145-170.

[46] J. Feldman, 'The simplicity principle in perception and cognition', WIREs Cognitive Science 7 (2016) 330-340.

[47] C. Freksa, 'Spatial cognition: an AI perspective', Proc. 16th European Conf. Artificial Intelligence (ECAI, Valencia, 2004), IOS Press 2004, pp. 1122-1128.

[48] C. Freksa, C. Habel and K. F. Wender (eds.), Spatial Cognition - an Interdisciplinary Approach to Representation and Processing of Spatial Knowledge, Lecture Notes in Computer Science 1404, Springer-Verlag, 1998.

[49] J. Fürnkranz, T. Kliegr and H. Paulheim, 'On cognitive preferences and the plausibility of rule-based models', Machine Learning 109 (2020) 853-898.

[50] N. Gauvrit, H. Singmann, F. Soler-Toscano and H. Zenil, 'Algorithmic complexity for psychology: a user-friendly implementation of the coding theorem method', Behavior Research Methods 48 (2016) 314-329.

[51] F. Gobet, P. C. R. Lane, S. Croker, P. C-H. Cheng, G. Jones, I. Oliver and J. M. Pine, 'Chunking mechanisms in human learning', Trends in Cognitive Sciences 5 no. 6 (2001) 236-243.

[52] F. Gobet and H. Simon, 'Expert chess memory: revisiting the chunking hypothesis', Memory 6 (1998) 225-255.

[53] O. Grabar, 'The intermediary of geometry', chapter 3 of The Mediation of Ornament, Princeton Univ. Press, 1989, pp. 119-154.

[54] L. K. M. Graf and J. R. Landwehr, 'A dual-process perspective on fluency-based aesthetics: the pleasure-interest model of aesthetic liking', Personality and Social Psychology Review 19 (2015) 395-410.

[55] L. K. M. Graf and J. R. Landwehr, 'Aesthetic pleasure versus aesthetic interest: the two routes to aesthetic liking', Frontiers in Psychology 8 (2017) article 15.

[56] R. M. Granovskaya, I. Ya. Bereznaya and A. N. Grigorieva, Perception of Form and Forms of Perception, Erlbaum, 1987. 
[57] V. Graziano, T. Glasmachers, T. Schaul, L. Pape, G. Cuccu, J. Leitner and J. Schmidhuber, 'Artificial curiosity for autonomous space exploration', Acta Futura 4 (2011) $41-51$.

[58] A. G. Greenwald and M. R. Banaji, 'The implicit revolution: reconceiving the relation between conscious and unconscious', American Psychologist 72 no. 9 (2017) 861-871.

[59] B. Grünbaum and G. C. Shephard, Tilings and Patterns, W. H. Freeman, 1987.

[60] B. Hampe (ed.), From Perception to Meaning: Image Schemas in Cognitive Linguistics, Cognitive Linguistics Research 29, Mouton de Gruyter, 2005.

[61] G. Hatfield, Perception and Cognition: Essays in the Philosophy of Psychology, Oxford Univ. Press, 2009.

[62] G. Hatfield and W. Epstein, 'The status of the minimum principle in the theoretical analysis of visual perception', Psychological Bulletin 97 no. 2 (1985) 155-186.

[63] D. Hilbert, Grundlagen der Geometrie, Teubner, Leipzig, 1899.

English translation: E. J. Townsend, The Foundations of Geometry, Open Court Publ. Co., 1902.

[64] J. Hochberg, 'Acts of perceptual inquiry: problems for any stimulus-based simplicity theory', Acta Psychologica 114 (2003) 215-228.

[65] J. E. Hochberg and V. Brooks, 'The psychophysics of form: reversible perspective drawings of spatial objects', American J. Psychology 73 (1960) 337-354.

[66] J. E. Hochberg and E. McAlister, 'A quantitative approach to figural "goodness"', J. Experimental Psychology 46 (1953) 361-364.

[67] C. S. Kaplan, 'Islamic star patterns from polygons in contact', Graphics Interface 2005, ACM International Conference Proceeding Series 112, 2005, pp. 177-186.

[68] M. Kharazmi, 'A study on geometric constructions on brickwork decorations in Iranian architecture', Proc. Bridges: Mathematics, Music, Art, Architecture, Education, Culture, (Jyväskylä, Finland, 2016), eds. E. Torrence, B. Torrence, C. H. Séquin, D. McKenna, K. Fenyvesi and R. Sarhangi, 2016, pp. 301-308.

[69] R. Kimchi and M. Goldsmith, 'Structure and process in perceptual organization', Advances in Psychology 93 (1992) 77-105.

[70] V. Kreinovich and L. Longpré, 'Human visual perception and Kolmogorov complexity: revisited', Bulletin European Assoc. Theoretical Computer Science 64 (1998) 155-158.

[71] B. Landau and R. Jackendoff, "What" and "where" in spatial language and spatial cognition', Behavioral and Brain Sciences 16 (1993) 217-265.

[72] E. L. J. Leeuwenberg, Structural Information of Visual Patterns: an Efficient Coding System in Perception, Mouton, 1967. 
[73] M. Li and P. M. B. Vitányi, An Introduction to Kolmogorov Complexity and its Applications (2nd edition). Springer, 1997.

[74] E. Mach, Space and Geometry in the Light of Physiological, Psychological and Physical Inquiry, translated by T. J. McCormack, Open Court Publ. Co., 1960.

[75] E. Mach, The Analysis of Sensations and the Relation of the Physical to the Psychical, translated by C. M. Williams, Open Court Publ. Co., 1984.

[76] P. Maguire, P. Moser, R. Maguire and M. T. Keane, 'Seeing patterns in randomness: a computational model of surprise', Topics in Cognitive Science 11 (2019) 103-118.

[77] E. Makovicky, '800-year old pentagonal tiling from Maragha, Iran, and the new varieties of aperiodic tiling it inspired', Fivefold Symmetry, ed. I. Hargittai, World Scientific, 1992, pp. 67-86.

[78] I. C. Mammarella, E. Borella, M. Pastore and F. Pazzaglia, 'The structure of visuospatial memory in adulthood', Learning and Individual Differences 25 (2013) 99-110.

[79] I. C. Mammarella, F. Pazzaglia and C. Cornoldi, 'Evidence for different components in children's visuospatial working memory', British J. Developmental Psychology 26 (2008) 337-355.

[80] J. M. Mandler and C. P. Cánovas, 'On defining image schemas', Language and Cognition 6 (2014) 510-532.

[81] J. L. Marroquin, Human Visual Perception of Structure, M.Sc. Thesis, MIT, 1976.

[82] D. E. Melnikoff and J. A. Bargh, 'The mythical number two', Trends in Cognitive Sciences 22 (2018) 280-293.

[83] C. Meneghetti, E. Labate, F. Pazzaglia, C. Hamilton and V. Gyselinck, 'The role of visual and spatial working memory in forming mental models derived from survey and route descriptions', British J. Psychology 108 no. 2 (2016) 225-243.

[84] S. V. Meyler, 'The phenomenon of ambiguity in art', chapter 1 in Insights into Perceptual Ambiguity and Inference in Art - a Practice-based Approach Derived from the Corporeal Form, Ph.D. Thesis, Univ. Lisbon, 2018.

[85] G. A. Miller, 'The magical number seven, plus or minus two: some limits on our capacity for processing information', Psychological Review 63 no. 2 (1956) 81-97.

[86] A. Morgan, 'Representations gone mental', Synthese 191 no. 2 (2014) 213-244.

[87] C. Muth, V. M. Hesslinger and C.-C. Carbon, 'The appeal of challenge in the perception of art: how ambiguity, solvability of ambiguity, and the opportunity for insight affect appreciation', Psychology of Aesthetics, Creativity, and the Arts 9 (2015) 206-216.

[88] A. Norenzayan, I. Choi and K. Peng, 'Perception and cognition', Handbook of Cultural Psychology, eds. S. Kitayama and D. Cohen, Guilford Press, 2007, pp. 569-594. 
[89] U. H. Obaidellah, The Role of Chunking and Schemas in Learning from Drawing, Ph.D. Thesis, Univ. Sussex, 2012.

[90] S. E. Palmer, 'Fundamental aspects of cognitive representation', Cognition and Categorization, eds. E. Rosch and B. Lloyd, Lawrence Elbaum Associates, 1978, pp. 259-303.

[91] F. Pazzaglia and R. De Beni, 'Strategies of processing spatial information in survey and landmark-centred individuals', European J. Cognitive Psychology 13 no. 4 (2001) 493-508.

[92] D. Pitt, 'Mental representation', The Stanford Encyclopedia of Philosophy, ed. E. N. Zalta, Spring 2020.

https://plato.stanford.edu/archives/spr2020/entries/mental-representation/

[93] J. R. Pomerantz and M. Kubovy, 'Theoretical approaches to perceptual organization: simplicity and likelihood principles', in Handbook of Perception and Human Performance, Vol. 2. Cognitive Processes and Performance eds. K. R. Boff, L. Kaufman and J. P. Thomas, John Wiley and Sons, Oxford, 1986, pp. 1-46.

[94] D. Reilly and D. L. Neumann, 'Gender-role differences in spatial ability: a meta-analytic review', Sex Roles 68 no. 9 (2013) 521-535.

[95] D. E. Rumelhart and D. A. Norman, 'Representation in memory', Stevens' Handbook of Experimental Psychology: Perception and Motivation; Learning and Cognition, eds. R. C. Atkinson, R. J. Herrnstein, G. Lindzey, and R. D. Luce, John Wiley and Sons, 1988, pp. 511-587.

[96] S. E. Schaeffer, 'Graph clustering', Computer Science Review 1 (2007) 27-64.

[97] T. Schaul, L. Pape, T. Glasmachers, V. Graziano and J. Schmidhuber 'Coherence progress: a measure of interestingness based on fixed compressors', Artificial General Intelligence, Proc. Fourth Intern. Conf. AGI 2011, eds J. Schmidhuber, K. R. Thorisson and M. Looks, Lecture Notes in Artificial Intelligence 6830, Springer-Verlag, 2011, pp. 21-30.

[98] M. Scheutz, 'The ontological status of representations', Understanding Representation in the Cognitive Sciences, eds. A. Riegler, M. Peschl and A. von Stein, Plenum, 1999, pp. 33-38.

[99] J. Schmidhuber, 'Simple algorithmic theory of subjective beauty, novelty, surprise, interestingness, attention, curiosity, creativity, art, science, music, jokes', J. Soc. Instrument and Control Engineers 48 no. 1 (2009) 21-32.

[100] J. Schmidhuber, 'Driven by compression progress: a simple principle explains essential aspects of subjective beauty, novelty, surprise, interestingness, attention, curiosity, creativity, art, science, music, jokes', Anticipatory Behavior in Adaptive Learning Systems. From Psychological Theories to Artificial Cognitive Systems, eds. G. Pezzulo, M. V. Butz, O. Sigaud, and G. Baldassarre, Lecture Notes in Computer Science 5499, Springer-Verlag, 2009, pp. 48-76.

[101] N. Shea, Representation in Cognitive Science, Oxford Univ. Press, 2018. 
[102] A. W. Siegel and S. H. White, 'The development of spatial representations of largescale environments', Advances in Child Development 10, ed. H. W. Reese, Academic Press, 1975, pp. 37-55.

[103] P. J. Silvia, 'What is interesting? Exploring the appraisal structure of interest', Emotion 5 no. 1 (2005) 89-102.

[104] P. J. Silvia, 'Cognitive appraisals and interest in visual art: exploring an appraisal theory of aesthetic emotions', Empirical Studies of the Arts 23 no. 2 (2005) 119-133.

[105] P. J. Silvia, 'Emotional responses to art: from collation and arousal to cognition and emotion', Review of General Psychology 9 no. 4 (2005) 342-357.

[106] P. J. Silvia, R. A. Henson and J. L. Templin, 'Are the sources of interest the same for everyone? Using multilevel mixture models to explore individual differences in appraisal structures', Cognition and Emotion 23 no. 7 (2009) 1389-1406.

[107] I. Spence and J. Feng, 'Video games and spatial cognition', Review of General Psychology 14 no. 2 (2010) 92-104.

[108] K. E. Stanovich, 'Distinguishing the reflective, algorithmic, and autonomous minds: is it time for a tri-process theory?', In Two Minds: Dual Processes and Beyond, eds. J. St. B. T. Evans and K. Frankish, Oxford Univ. Press, 2009, pp. 55-88.

[109] P. Suppes, M. Pavel and J.-Cl. Falmagne, 'Representations and models in psychology', Annual Review of Psychology 45 (1994) 517-544.

[110] N. Tinbergen, 'Über die Orientierung des Bienenwolfes (Philantus triangulum Fabr.)', Zeitschrift für vergleichende Physiologie 16 (1932) 305-335.

[111] N. Tinbergen and W. Kruyt, 'Über die Orientierung des Bienenwolfes (Philantus triangulum Fabr.)', Zeitschrift für vergleichende Physiologie 25 (1938) 292-334.

[112] L. Tommasi, C. Chiandetti, T. Pecchia, V. A. Sovrano and G. Vallortigara, 'From natural geometry to spatial cognition', Neuroscience and Biobehavioral Reviews 36 (2012) 799-824.

[113] J. Trilling, The Language of Ornament, Thames and Hudson, 2001.

[114] G. van Beusekom, 'Some experiments on the optical orientation in Philantus triangulum Fabr.', Behaviour 1 (1948) 195-225.

[115] S. van de Cruys and J. Wagemans, 'Putting reward in art: a tentative prediction error account of visual art', i-Perception 2 (2011) 1035-1062.

[116] P. A. van der Helm, 'Simplicity in perceptual organization', Oxford Handbook of Perceptual Organization, ed. J. Wagemans, Oxford Univ. Press, 2015, pp. 1027-1045.

[117] P. A. van der Helm, R. J. van Lier and E. L. J. Leeuwenberg, 'Serial pattern complexity: irregularity and hierarchy', Perception 21 (1992) 517-544. 
[118] G. Westphal-Fitch, L. Huber, J. C. Gómez and W. T. Fitch, 'Production and perception rules underlying visual patterns: effects of symmetry and hierarchy', Philosophical Trans. Royal Soc. Series B 367 (2012) 2007-2022.

[119] B. Wood, 'Pleasure and meaning in Islamic art: toward a naturalist framework', $J$. Utah Academy Sciences, Arts, and Letters 97 (2020) 47-63.

[120] W. Wundt, Grundzüge der Physiologischen Psychologie, Engelmann, Leipzig, 1874.

[121] H. Zenil, N. A. Kiani and J. Tegnér, 'Symmetry and correspondence of algorithmic complexity over geometric, spatial and topological representations', Entropy 20 (2018) article 534 .

[122] H. Zenil, N. A. Kiani and J. Tegnér, 'A review of graph and network complexity from an algorithmic information perspective', Entropy 20 (2018) article 551.

[123] H. Zenil, F. Soler-Toscano, J.-P. Delahaye and N. Gauvrit, 'Two-dimensional Kolmogorov complexity and an empirical validation of the Coding theorem method by compressibility', PeerJ Computer Science 1 (2015) article cs-23. 


\section{Appendix}

In $\S 8$ we posed the problem of converting a geometric diagram that furnished a structural representation of a pattern into a sequence of geometric operations to obtain an equivalent constructive representation. The diagrams in Figure 17 provide a solution to this puzzle and break down the construction of Figure 7(b) into the following steps.

(a) Take a square in which to construct the template. Draw the diagonal $O A$. Draw a circular arc centred at $O$ through corner $B$. Let $C$ be the point where the arc crosses the diagonal. The details of the rest of the construction will be concentrated in the top-left triangle $O A B$; some of the lines are reflected into the bottom-right triangle without comment.

(b) Draw a circular arc centred at $A$ through the point $C$. Bisect the angle $\measuredangle A O B$ to obtain the line $O D$. Draw the line segment $C D$.

(c) Bisect the angle $\measuredangle O D B$ to obtain the line $D E$. Bisect the angle $\measuredangle O D C$ to obtain the line $D F$.

(d) The line through $B$ and $C$ contains the first parts of the design (shown as the solid black lines); it meets $D E$ at the point $G$. The line $E F$ meets the line $O D$ at the point $H$. The circular arc centred at $O$ through the point $F$ carries the information into the lower triangle by marking the point on the bottom side of the square.

(e) Draw a line through $G$ and $H$ until it meets the line $O I$. Let $J$ be the point where it crosses the line $O A$, and let $K$ be the point where it meets $O I$. Draw circular arcs centred at $O$ through the points $J$ and $K$.

(f) The construction lines now provide the locations of all the points needed to complete the design.

This illustrates that using algorithms as a model for mental representations should take the difficulty of production into account, not only the length of the algorithm. 


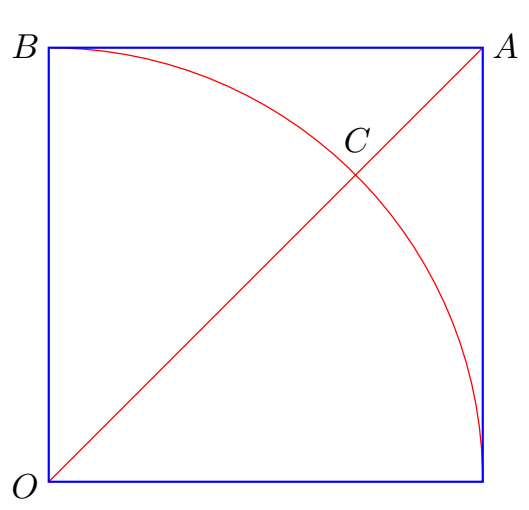

(a)

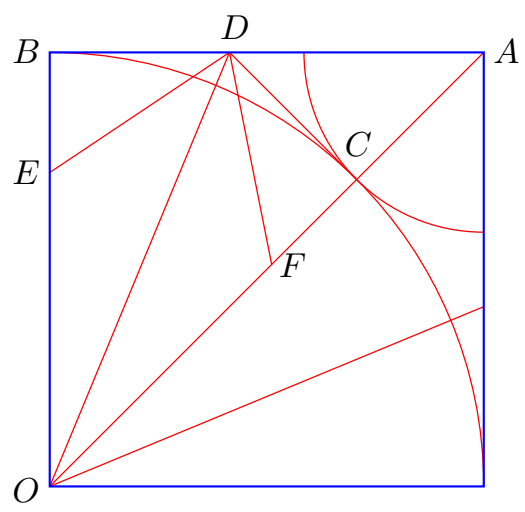

(c)

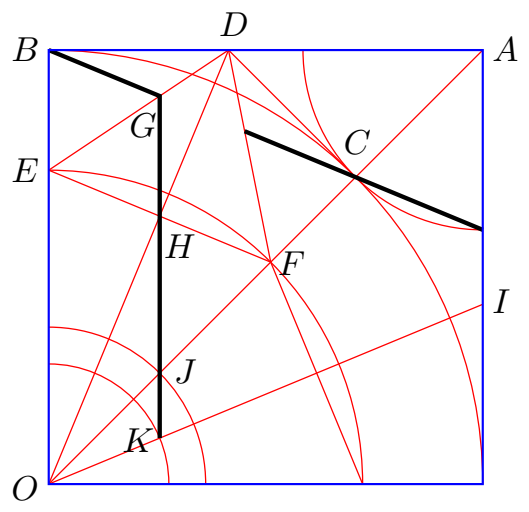

(e)

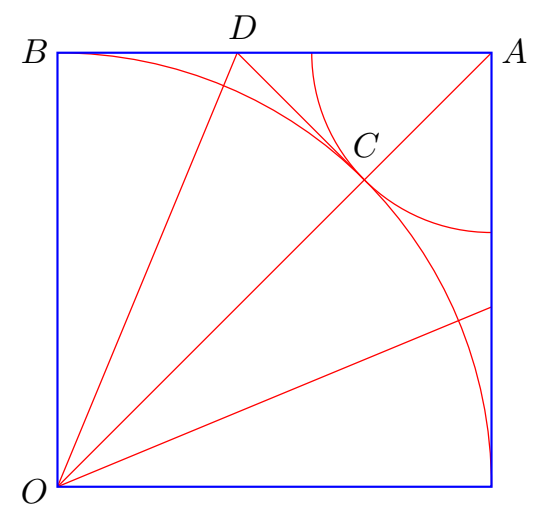

(b)

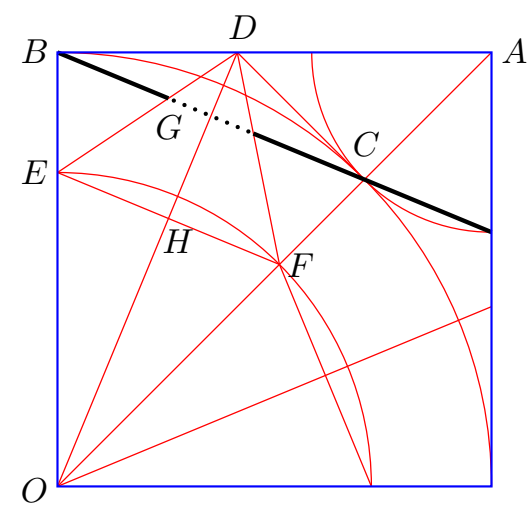

(d)

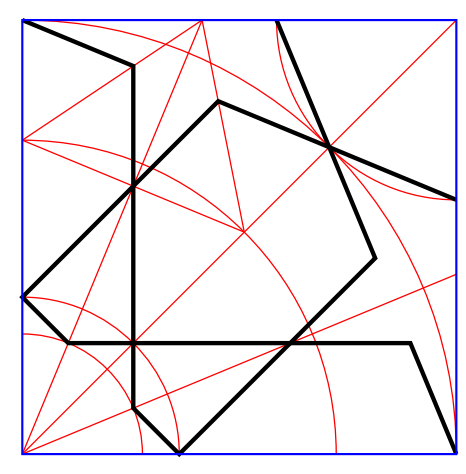

(f)

Figure 17: Deconstruction of a diagram into a sequence of simple geometric operations. 